\title{
Covariant description for frictional contact problems
}

Alexander Konyukhov, Karl Schweizerhof Universität Karlsruhe, Institut für Mechanik

Institut für Mechanik

Kaiserstr. 12, Geb. 20.30

76128 Karlsruhe

Tel.: +49 (0) 721/ 608-2071

Fax: +49 (0) 721/ 608-7990

E-Mail: ifm@uni-karlsruhe.de

www.ifm.uni-karlsruhe.de 


\title{
Covariant description for frictional contact problems
}

\author{
A. Konyukhov, K. Schweizerhof
}

2005

\begin{abstract}
A fully covariant description, based on the consideration of contact from the surface geometry point of view, is used for a consistent formulation of frictional contact conditions. All necessary operations for the description of the contact problems: kinematics, all differential operations etc. are defined in the covariant form in the local coordinate system which corresponds to the closest point procedure. The main advantage is a geometrical structure of the full tangent matrix, which is is subdivided into main, rotational and curvature parts. The consistent linearization of the penalty regularized contact integral leads to a symmetrical tangent matrix in the case of sticking. Representative examples show the effectiveness of the approach for problems where the definition of sticking-sliding zones is necessary as well as for the case of fully developed sliding zones.
\end{abstract}

\section{Keywords}

Frictional contact problem, covariant description, Tangent matrix, sticking, sliding, evolution equations.

\section{Introduction}

With frictional contact a specific interaction between bodies contacting each other along surfaces of those bodies is described. Differential geometry provides a powerful mathematical tool to capture the change of these surfaces in the covariant form. Another essential feature to model frictional contact problems is the formulation of the contact conditions as kinematical constraints which leads to a nonlinear problem and, therefore, in the correct description of the solution process, to a consistent linearization problem. The Lagrange multiplier method as well as various regularization techniques are among the solutions schemes available to satisfy the contact conditions. E. g. for 2D frictional problems Wriggers et. al. [28] used the elasto-plastic analogy and the penalty regularization of contact conditions. By then the return mapping algorithm developed for the plasticity problem was linearized in the global coordinate system. Peric and Owen [20] used the penalty method for 3D frictional contact problems with small deformations. Laursen and Simo [15], however, formulated the penalty based contact conditions and the return mapping algorithm via convective surface coordinates, but the following linearization performed in the global coordinate system led to an artificial non-symmetry of the tangent matrix in the case of sticking. The symmetrization based on the nested Augmented Lagrangian algorithm was proposed in Simo and Laursen [24] to gain back the symmetry of the tangent matrices, but this is not a consistent procedure. Pietrzak and Curnier [21] worked extensively with the Augmented Lagrangian formulation, which was still formulated in global coordinates though with an usage of the convective coordinates. Parish and Lübbing [19] used also the convective conditions together with the penalty regularization for sticking and sliding, but still obtain a non-symmetric stick tangent matrix. Wriggers in [31] mentions the regularization of the stick conditions based on a functional used in mesh tying procedures which consequently leads to a symmetric tangent matrix. An alternative approach preserving symmetry in 2D for sticking, based on the so-called moving cone was proposed in Krstulovic-Opara and Wriggers [12]. Another problems arises from 
the artificial non-smoothness of the contact surfaces modeled by low-order polynomial functions leading to oscillations of the major characteristics of the solution. Various techniques based on smooth approximations of contact surfaces can be found in [16], [4], [1], [21]. Wriggers et. al. [30] mentioned e.g. a problem concerning the discontinuity of the history variables at element boundaries for smooth surfaces and proposed to use the path length in the 2D case. Various techniques based on geometrical forms in global coordinates were later considered for 3D problems in Krstulovic-Opara et. al. [13] and in Puso and Laursen [22].

Despite the large amount of contributions the fully covariant description of contact is still not available in literature. In this contribution we employ the highly developed "apparatus" of differential geometry (see e.g. Gray [5]) to reconsider the contact conditions in a specially defined spatial local coordinate system which corresponds to the well-known closest point procedure. All differential operations necessary for kinematics and linearization are considered as covariant derivatives (see Marsden and Hughes [18]). Special attention is on the consideration of the operations and the weak form on the tangent plane. The constitutive equations for the tangential tractions within the penalty regularization, or, so called, the evolution equations, are considered in the covariant description as a parallel translation on the contact surface. It is important to use this form of the constitutive equations, because the consistent linearization of the contact integral together with these equations leads to a symmetrical tangent matrix on the tangent plane in the case of sticking. Each part of the full tangent matrix, such as the normal tangent matrix, the tangent matrix in the case of sticking and the tangent matrix in the case of sliding has a geometrical structure, and, in due course, is subdivided into main, rotational and curvature parts. In addition the geometrical interpretation of the parallel translation allows to develop an integration scheme for the tangential tractions and to overcome the problem of the discontinuity of the history variables at element boundaries. The frictional contact problem can be subdivided for numerical solutions into two types depending on the necessity to capture the stick-slip behavior precisely by considering the numerical integration of the evolution equations. The "segment-to-segment", the "node-tosegement" and the "segment-to-analytical surface" finite element approaches are considered and discussed for different types of contact problems.

The article is organized as follows. In the first section of the part "Geometry and Kinematics of Contact" we recall all the operations necessary for our development, known from differential geometry. The core of the contribution is the second section where a spatial coordinate system corresponding to the closest point projection procedure is built. Kinematics of contact and differential operations are revisited in this coordinate system. In the third section the numerical algorithms to compute the characteristics from the geometrical point of view are presented. In particular the weak form, the penalty regularization and the return-mapping algorithm are considered with a special attention on the construction of the evolution equations for the tangent tractions. The developed equations are combined during the linearization in the fourth section. The fifth section contains a summary of the results which are necessary for finite element implementation. A series of the numerical examples shows the effectiveness of the proposed technique in the sixth section.

\section{Geometry and Kinematics of Contact}

We consider two interacting bodies (Figure 1). One of them is chosen as the contact body: its surface is called "master" surface. On the surface of the second body, we consider a "slave" point $S$, which is e.g. an integration or a nodal point. Two bodies are coming into contact, if a slave point of the second surface penetrates into the master surface, where penetration is defined as the shortest distance between the two surfaces of the contacting bodies. 


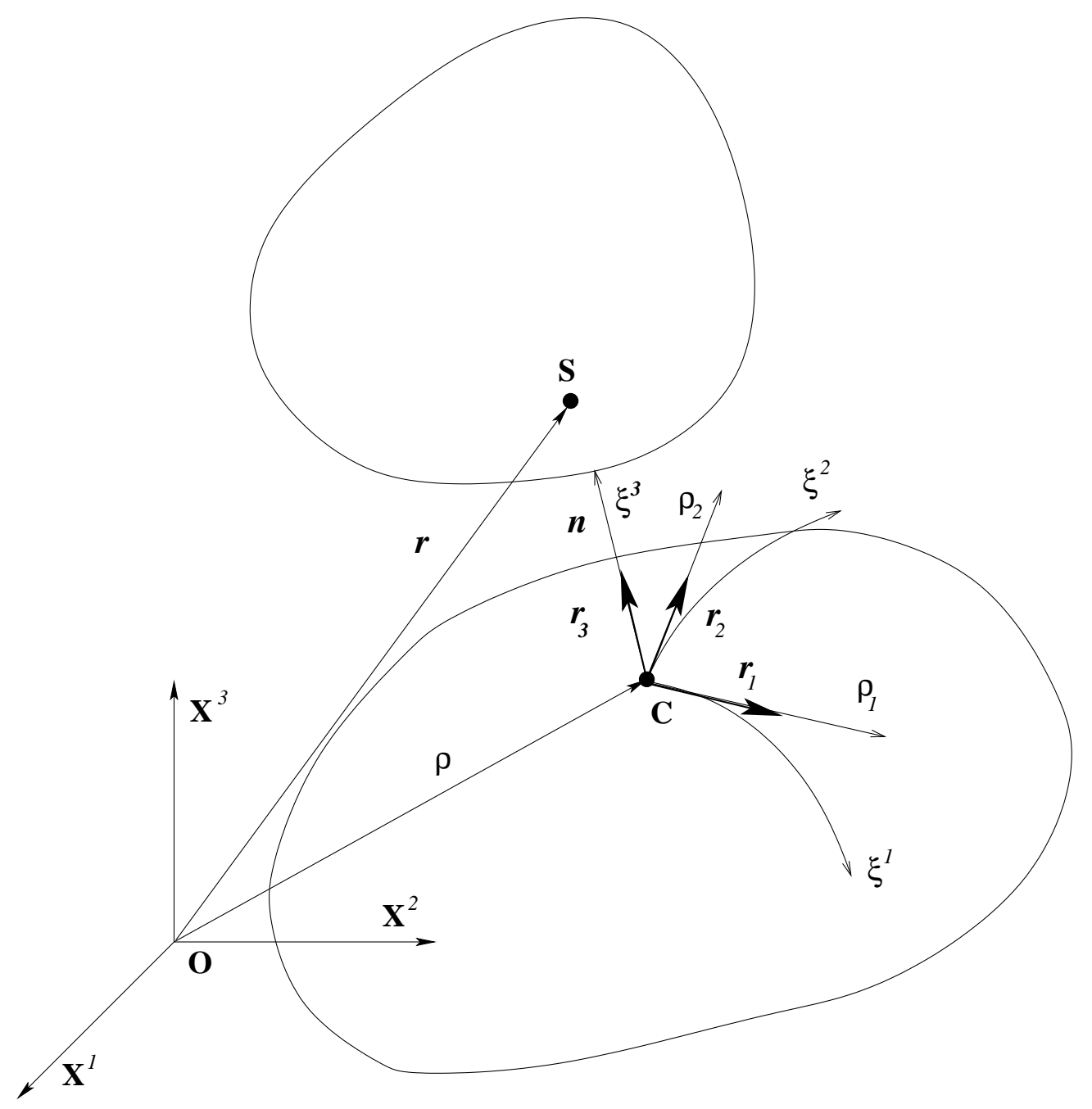

Figure 1: Two body contact. Local surface coordinate system on master surface.

As contact between two bodies is dominantly an interaction between these two surfaces, the main aim of the following consideration is to take advantage of the differential geometry of the contact surfaces in order to describe the kinematics of the contact conditions. First, we consider the geometry of the master surface and its characteristics and then define a special spatial coordinate system attached to this surface.

\subsection{Local surface coordinate system and its geometrical characteristics}

The "master" surface of the body (Fig. 1), is a 2D manifold, and therefore, can be parameterized by the surface coordinates $\xi^{1}, \xi^{2}$. Let $\boldsymbol{\rho}$ be a surface vector, describing any point on the surface. In a finite element discretization this can be done in the following form

$$
\boldsymbol{\rho}=\sum_{k} N_{k}\left(\xi^{1}, \xi^{2}\right) \mathbf{x}^{(k)},
$$

where $N_{k}\left(\xi^{1}, \xi^{2}\right)$ are shape functions and $\mathbf{x}^{(k)}$ are nodal coordinates. The set of shape functions can be either of the same order as for the finite discretization of the contact body, or it can be constructed differently as for the case of the smooth approximation of the contact surfaces. It must be noted that the parameterization (1) is locally defined on the surface element. Therefore, 
the internal variables $\xi^{i}$ are not continuous between the boundaries of adjacent surface elements. This leads to a problem for the kinematical characteristics while crossing the element boundaries.

We consider here quasi-static problems, therefore, we assume that $\mathbf{x}^{(k)}=\mathbf{x}^{(k)}(t)$, where time is treated as a load increment parameter. In general, we consider the geometry of moving surfaces. A specific focus is on the solution scheme, for which the nonlinear equations have to be linearized. Within the velocity description the increment vector is then treated as a velocity vector.

\subsubsection{The fundamental tensors and property of the contact surface}

Two fundamental tensors of the surface: the metrics tensor, or the first fundamental tensor, and the curvature tensor, or the second fundamental tensor, fully contain the properties of a surface. The metrics tensor is responsible for any metric operation on the surface (length, area or angle). The curvature tensor is responsible for the inclusion of a surface as a 2D manifold into the 3D space $\boldsymbol{R}^{3}$ (Cartesian space) respectively for the local structure of the surface in the 3D space.

First, two surface base vectors $\boldsymbol{\rho}_{i}, \quad i=1,2$ in the tangent plane of a surface are introduced

$$
\boldsymbol{\rho}_{1}=\frac{\partial \boldsymbol{\rho}}{\partial \xi^{1}}, \quad \boldsymbol{\rho}_{2}=\frac{\partial \boldsymbol{\rho}}{\partial \xi^{2}}
$$

then the normal unit surface vector is given as the cross product of the basis vectors

$$
\mathbf{n}=\frac{\boldsymbol{\rho}_{1} \times \boldsymbol{\rho}_{2}}{\left|\boldsymbol{\rho}_{1} \times \boldsymbol{\rho}_{2}\right|} .
$$

These three vectors $\boldsymbol{\rho}_{1}, \boldsymbol{\rho}_{2}, \mathbf{n}$ define a local surface coordinate system and they are used to obtain the two fundamental tensors of the surface [5], [23].

The metric tensor. The covariant components of the metric tensor on the surface are defined as the dot product of the base surface vectors (2)

$$
a_{i j}=\boldsymbol{\rho}_{i} \cdot \boldsymbol{\rho}_{j}, \quad i, j=1,2
$$

The contravariant components of the metric tensor $a^{i j}$ are obtained via the equation

$$
a_{i k} a^{k j}=a_{i .}^{\cdot j}=\delta_{i}^{j},
$$

i. e. as the inverse matrix is given in the following form:

$$
a^{i j}: \quad \frac{1}{a}\left[\begin{array}{cc}
a_{22} & -a_{12} \\
-a_{12} & a_{11}
\end{array}\right], \quad a=\operatorname{det}\left(a_{i j}\right)=a_{11} a_{22}-\left(a_{12}\right)^{2},
$$

the mixed components $a_{i .}^{j}$ are in fact identical to the Kronecker delta $\delta_{i}^{j}$.

An adjacent basis of the surface is defined by the contravariant base vectors $\boldsymbol{\rho}^{i}$, which are obtained via a linear form of the covariant base vectors:

$$
\boldsymbol{\rho}^{i}=a^{i j} \boldsymbol{\rho}_{j} .
$$

Thus, the metric tensor can be defined either in the covariant, or the contravariant basis, or the mixed basis

$$
\mathbf{A}=a^{i j} \boldsymbol{\rho}_{i} \otimes \boldsymbol{\rho}_{j}=a_{i j} \boldsymbol{\rho}^{i} \otimes \boldsymbol{\rho}^{j}=a_{i .}^{. j} \boldsymbol{\rho}^{i} \otimes \boldsymbol{\rho}_{j} .
$$


The metrical characteristics, which are necessary for the further description, are length and area. The differential $d l$ of the length is obtained as

$$
d l=\sqrt{\left(\boldsymbol{\rho}_{i} \cdot \boldsymbol{\rho}_{j}\right) d \xi^{i} d \xi^{j}}=\sqrt{a_{i j} d \xi^{i} d \xi^{j}} .
$$

The differential $d s$ of the area can be obtained either via the determinant $a$ of the matrix of the metric tensor eqn. (4), or via the absolute value of the cross product of the surface vectors eqn. (3):

$$
d s=\sqrt{\left|\operatorname{det}\left(a_{i j}\right)\right| d} \xi^{1} d \xi^{2}=\left|\boldsymbol{\rho}_{1} \times \boldsymbol{\rho}_{2}\right| d \xi^{1} d \xi^{2} .
$$

The curvature tensor. In differential geometry the curvature tensor is used to describe a local surface structure via e.g. main curvatures, for more information see [5], [23]. The tensor is defined by its covariant components $h_{i j}$, which are computed as the dot product of the second derivative of the vector $\boldsymbol{\rho}$ and the normal $\mathbf{n}$

$$
h_{i j}=\boldsymbol{\rho}_{i j} \cdot \mathbf{n} .
$$

The contravariant components are obtained as a bilinear combination of the covariant components with the contravariant metrics components

$$
h^{i j}=h_{k n} a^{i k} a^{n j} .
$$

Equation (12) gives also a general rule how to compute contravariant components of any second order tensor via covariant components.

\subsubsection{Differential operations in the surface coordinate system}

For any further derivations the most important mathematical operations in the surface coordinate system are frame independent differential operations. They are defined in order to describe the kinematics from the local surface coordinate system point of view. For this the derivatives of base vectors have to be determined. The Weingarten formula and the Gauss-Kodazzi formula [23] give us a complete set for derivatives of base vectors and are used to define covariant derivatives on the surface.

The Weingarten formula gives directly derivatives of the unit normal-prove see Appendix A:

$$
\mathbf{n}_{i}=-h_{i j} a^{j k} \boldsymbol{\rho}_{k}=-h_{i}^{k} \boldsymbol{\rho}_{k} .
$$

The Gauss-Kodazzi formula allows directly the computation of derivatives of the basis vectors $\boldsymbol{\rho}_{i}$.

$$
\boldsymbol{\rho}_{i j}=\Gamma_{i j}^{k} \boldsymbol{\rho}_{k}+h_{i j} \mathbf{n}
$$

where $\Gamma_{i j}^{k}$ are Christoffel symbols [23], [18], defined on the surface as follows

$$
\Gamma_{i j}^{k}=\boldsymbol{\rho}_{i j} \cdot \boldsymbol{\rho}^{k}=\boldsymbol{\rho}_{i j} \cdot \boldsymbol{\rho}_{n} a^{n k} .
$$

For the prove see Appendix B. 
Indifferent covariant derivative. The derivatives of the base vectors allow to evaluate a frame indifferent derivative of any object defined on the surface. We now consider a vector $\mathbf{T}$, defined by its local contravariant coordinates $T^{i}$ in the surface coordinate system

$$
\mathbf{T}=T^{i} \boldsymbol{\rho}_{i} .
$$

The full time derivative of $\mathbf{T}$ with the assumption that the vector $\boldsymbol{\rho}_{i}$ is implicitly time dependent via the coordinates $\xi^{i}$ gives

$$
\frac{d}{d t} \mathbf{T}=\frac{\partial T^{i}}{\partial t} \boldsymbol{\rho}_{i}+\frac{\partial T^{i}}{\partial \xi^{j}} \dot{\xi}^{j} \boldsymbol{\rho}_{i}+T^{i} \dot{\xi}^{j} \boldsymbol{\rho}_{i j}
$$

Further applying the Gauss-Kodazzi formula, we get

$$
\frac{d}{d t} \mathbf{T}=\frac{\partial T^{i}}{\partial t} \boldsymbol{\rho}_{i}+\left(\frac{\partial T^{i}}{\partial \xi^{j}}+T^{k} \Gamma_{j k}^{i}\right) \dot{\xi}^{j} \boldsymbol{\rho}_{i}
$$

Finally, the full material time derivative of the vector has the following form

$$
\frac{d}{d t} \mathbf{T}=\left(\frac{\partial T^{i}}{\partial t}+\nabla_{j} T^{i} \dot{\xi}^{j}\right) \boldsymbol{\rho}_{i}
$$

The term $\nabla_{j} T^{i}$ is a covariant derivative of the contravariant component $T^{i}$

$$
\nabla_{j} T^{i}=\frac{\partial T^{i}}{\partial \xi^{j}}+T^{k} \Gamma_{j k}^{i}
$$

A similar expression can be found for the covariant derivative of the covariant components $T_{i}$, see prove in Appendix $\mathbf{C}$ :

$$
\nabla_{j} T_{i}=\frac{\partial T_{i}}{\partial \xi^{j}}-T_{k} \Gamma_{i j}^{k}
$$

\subsection{Spatial coordinate system and its characteristics}

As discussed above two bodies come into contact if a slave point penetrates at least at the closest distance into the master surface. This point is computed via the well known closest point procedure, see details for the finite element implementation in Wriggers [31], Laursen [17]. This procedure can be included in the variational formulation, see also the theoretical details in Kikuchi and Oden [9]. One of the important aspects in the current contribution is to construct a special spatial coordinate system on the master surface corresponding to the projection procedure and to consider then the contact integral as well as a linearization procedure in this system.

\subsubsection{Projection of the contact point vector onto the master surface.}

We recall here the projection procedure with specific attention on the definition of all necessary parameters via the surface characteristics. At the location $C$ on the surface described by the vector $\boldsymbol{\rho}\left(t, \xi_{1}, \xi_{2}\right)$ (see Fig. 1), the value of the penetration of a surface into another one is defined as the minimal distance between these surfaces, see Kikuchi and Oden [9], Wriggers [31], Laursen [17]. This leads to the following extremal problem:

$$
\left\|\left(\mathbf{r}_{s}-\boldsymbol{\rho}\right)\right\| \rightarrow \min , \quad \longrightarrow\left(\mathbf{r}_{s}-\boldsymbol{\rho}\right) \cdot\left(\mathbf{r}_{s}-\boldsymbol{\rho}\right) \rightarrow \min
$$


As is well known, the solution of eqn. (21) can be achieved by the application of a Newton procedure for the function

$$
\mathbf{F}\left(\xi^{1}, \xi^{2}\right)=\left(\mathbf{r}_{s}-\boldsymbol{\rho}\right)^{2} .
$$

The convective coordinates $\xi_{n+1}^{i}$ at the penetration location $C$ are computed with the Newton scheme for the iteration $n+1$

$$
\begin{aligned}
\Delta \boldsymbol{\xi}_{n}=\left[\begin{array}{c}
\Delta \xi_{n+1}^{1} \\
\Delta \xi_{n+1}^{2}
\end{array}\right]=-\left(\mathbf{F}^{\prime \prime}\right)_{n}^{-1} \mathbf{F}_{n}^{\prime} & \\
& \boldsymbol{\xi}_{n+1}=\boldsymbol{\xi}_{n}+\Delta \boldsymbol{\xi}_{n},
\end{aligned}
$$

where the first derivative $\mathbf{F}^{\prime}$ and the second derivative $\mathbf{F}^{\prime \prime}$ with respect to the surface coordinates are described via the surface characteristics as:

$$
\begin{aligned}
& \mathbf{F}^{\prime}=\left[\begin{array}{c}
\frac{\partial F}{\partial \xi^{1}} \\
\frac{\partial F}{\partial \xi^{2}}
\end{array}\right]=-2 \cdot\left[\begin{array}{l}
\boldsymbol{\rho}_{1} \cdot\left(\mathbf{r}_{s}-\boldsymbol{\rho}\right) \\
\boldsymbol{\rho}_{2} \cdot\left(\mathbf{r}_{s}-\boldsymbol{\rho}\right)
\end{array}\right] \\
& \mathbf{F}^{\prime \prime}=2 \cdot\left[\begin{array}{ll}
a_{11}-\boldsymbol{\rho}_{11}\left(\mathbf{r}_{s}-\boldsymbol{\rho}\right) & a_{12}-\boldsymbol{\rho}_{12}\left(\mathbf{r}_{s}-\boldsymbol{\rho}\right) \\
a_{21}-\boldsymbol{\rho}_{22}\left(\mathbf{r}_{s}-\boldsymbol{\rho}\right) & a_{22}-\boldsymbol{\rho}_{22}\left(\mathbf{r}_{s}-\boldsymbol{\rho}\right)
\end{array}\right],
\end{aligned}
$$

with the components of $\mathbf{F}_{n}^{\prime}$ and $\mathbf{F}_{n}^{\prime \prime}$ evaluated at state $n$.

\subsubsection{Spatial local coordinate system. Geometrical characteristics on the tangent plane.}

Now we define a special local coordinate system related to the master surface at the penetration point $C$. Any spatial vector in space can be defined as

$$
\mathbf{r}\left(\xi^{1}, \xi^{2}, \xi^{3}\right)=\boldsymbol{\rho}+\mathbf{n} \xi^{3} .
$$

By assuming the normal vector to be known, the projection procedure has already been taken into account into this consideration. The equilibrium equations for contact will now be formulated in the defined local coordinate system, but since contact is an interaction between surfaces then each necessary equation especially for the linearization will be considered on the tangent plane, i.e. at $\xi^{3}=0$. For this, we define all the geometrical and differential characteristics with special attention on their values on the tangent plane.

The penetration. A value of the penetration $g$, essential for formulation of the non-penetration conditions in the contact mechanics, see [31], [17], [9], is exactly the third coordinate in our surface coordinate system:

$$
\xi^{3}=g=\left(\mathbf{r}_{s}-\boldsymbol{\rho}\right) \cdot \mathbf{n} .
$$

In the spatial curvilinear coordinate system all the characteristics as metrics, covariant derivative etc. considered before can be defined. We consider only those which are necessary for the further development. The base vectors of the system are given as

$$
\mathbf{r}_{i}=\frac{\partial \mathbf{r}}{\partial \xi^{i}}=\boldsymbol{\rho}_{i}+\mathbf{n}_{i} \xi^{3}=\left(a_{i}^{k}-h_{i}^{k} \xi^{3}\right) \boldsymbol{\rho}_{k}, \quad i=1,2, \quad \mathbf{r}_{3}=\mathbf{n},
$$


where the Weingarten formula (13) and the first fundamental tensor in the mixed formulation (5) have been used to obtain a more compact formula. The covariant components of the metric tensor of the spatial coordinate system are defined via the dot product of vectors eqn. (28).

$$
g_{i j}=\left(\mathbf{r}_{i} \cdot \mathbf{r}_{j}\right)=a_{i j}-2 \xi^{3} h_{i j}+h_{i k} h_{j}^{k}\left(\xi^{3}\right)^{2}, \quad i=1,2 \quad g_{i 3}=0, \quad g_{33}=1 .
$$

Contravariant metric components $g^{i j}$, as well as contravariant base vectors $\mathbf{r}^{i}$ are defined in a similar fashion, eqn. (5), (7).

Time derivative of the covariant metrics components $a_{i j}$. During the forthcoming linearization it is essential to consider this procedure as a 3D process in the spatial coordinate system (26). Therefore, in general, derivatives also with respect to the third coordinate $\xi^{3}$ should be considered. Thus, time derivatives of the surface metric components $a_{i j}$ are calculated as values of the spatial metric components $g_{i j}$ on the tangent plane at $\xi^{3}=0$, namely

$$
\begin{gathered}
\frac{d a_{m n}}{d t}=\left.\frac{d g_{m n}}{d t}\right|_{\xi^{3}=0}=\left.\left[\frac{\partial}{\partial t}+\dot{\xi}^{j} \frac{\partial}{\partial \xi^{j}}+\dot{\xi}^{3} \frac{\partial}{\partial \xi^{3}}\right]\left(a_{m n}-2 \xi^{3} h_{m n}+h_{m k} h_{n}^{k}\left(\xi^{3}\right)^{2}\right)\right|_{\xi^{3}=0}= \\
=\left(\frac{\partial}{\partial t}+\dot{\xi}^{j} \frac{\partial}{\partial \xi^{j}}\right)\left(\boldsymbol{\rho}_{m} \cdot \boldsymbol{\rho}_{n}\right)-2 h_{m n} \dot{\xi}^{3}= \\
=\left(\mathbf{v}_{m} \cdot \boldsymbol{\rho}_{n}\right)+\left(\boldsymbol{\rho}_{m} \cdot \mathbf{v}_{n}\right)+\left(\Gamma_{m j}^{l}\left(\boldsymbol{\rho}_{l} \cdot \boldsymbol{\rho}_{n}\right)+\Gamma_{n j}^{l}\left(\boldsymbol{\rho}_{m} \cdot \boldsymbol{\rho}_{l}\right)\right) \dot{\xi}^{j}-2 h_{m n} \dot{\xi}^{3} .
\end{gathered}
$$

The Christoffel symbols appear in eqn. (30) due to the usage of the Kodazzi formula. All indices are running from 1 to 2 .

Time derivative of the contravariant metrics components $a^{i j}$. The time derivative of the contravariant component of the metric tensor $a^{i j}$ is obtained from the derivation of eqn. (5):

$$
\frac{d}{d t}\left(a^{i k} a_{k j}\right)=0 \quad \longrightarrow a_{k j} \frac{d a^{i k}}{d t}+a^{i k} \frac{d a_{k j}}{d t}=0 \quad \longrightarrow \frac{d a^{i k}}{d t}=-a^{i m} a^{n k} \frac{d a_{m n}}{d t} .
$$

Spatial Christoffel symbols. Covariant derivative on the tangent plane. In order to distinguish in the summation agreement a spatial object from the surface one, we will use capital letters, i.e. $I, J, \ldots=\{1,2,3\}$. Covariant derivatives in the spatial coordinate system require the spatial Christoffel symbols $\hat{\Gamma}_{I J}^{K}$. They are defined, similar to eqn. (15) but with the spatial base vectors $\mathbf{r}_{I}$, as $\hat{\Gamma}_{I J}^{K}=\left(\mathbf{r}^{K} \cdot \mathbf{r}_{I J}\right)$. The full time derivative in the spatial coordinate system in the form of eqn. (18), computed in convective coordinates $\xi^{I}$ via covariant derivatives either for contravariant components in eqn. (19) or for covariant components in eqn. (20), is a frame indifferent derivative. It coincides with the Lie time derivative definition $\mathcal{L}_{t}$ in the form

$$
\mathcal{L}_{t} \mathbf{T}:=\mathbf{F} \frac{d}{d t}\left(\mathbf{F}^{-1} \mathbf{T}\right)=\frac{d}{d t} \mathbf{T}
$$

where $\mathbf{F}$ is a push-forward and $\mathbf{F}^{-1}$ is a pull-back operator, see more in Bonet and Wood [3], Marsden and Hughes [18]. For the prove of formula (32) see Appendix D. The Lie time derivative is usually exploited for the linearization, therefore, the computation of the covariant derivatives will be employed for further linearization. In the further considerations we concentrate on the full time derivative on the tangent plane. Then values of the spatial Christoffel symbols on the surfaces $\left.\hat{\Gamma}_{I J}^{K}\right|_{\xi^{3}=0}$, i.e. if $\xi^{3}=0$, define a value of covariant derivatives for any spatial object on 
the tangent plane. It can be easily seen from their definition and the Weingarten formula that the following relations between the spatial and surface terms hold:

$$
\left.\hat{\Gamma}_{i j}^{k}\right|_{\xi^{3}=0}=\Gamma_{i j}^{k}, \quad i, j, k=1,2,
$$

$$
\left.\hat{\Gamma}_{i j}^{3}\right|_{\xi^{3}=0}=0
$$

$$
\left.\hat{\Gamma}_{3 j}^{k}\right|_{\xi^{3}=0}=-h_{j}^{k},
$$

where $\Gamma_{i j}^{k}$ are the surface Christoffel symbols (15) and $h_{j}^{k}$ are mixed components of the curvature tensor.

With the vector $\mathbf{T}$ in the tangent plane in covariant components, i.e.

$$
\mathbf{T}=\left.T_{i} \mathbf{r}^{i}\right|_{\xi^{3}=0}=T_{i} \boldsymbol{\rho}^{i}
$$

its full time derivative is computed employing the rules given in (17) and (20)

$$
\begin{gathered}
\frac{d T_{i}}{d t}=\frac{\partial T_{i}}{\partial t}+\left(\frac{\partial T_{i}}{\partial \xi^{J}}-\hat{\Gamma}_{I J}^{K} T_{K}\right)_{\xi^{3}=0} \dot{\xi}^{J} \longrightarrow \\
=\frac{\partial T_{i}}{\partial t}+\left(\frac{\partial T_{i}}{\partial \xi^{j}}-\Gamma_{i j}^{k} T_{k}\right) \dot{\xi}^{j}+h_{i}^{k} T_{k} \dot{\xi}^{3} .
\end{gathered}
$$

One should distinguish that the full time derivative with the surface Christoffel symbols in the form eqn. given in (17) and (20) can be applied to an object that belongs to the internal geometry of the surface, e.g. for $\dot{\xi}^{i}$; for the full time derivative of an arbitrary spatial object, positioned in the tangent plane, the form in eqn. (35) must be used.

\subsubsection{Motion of a slave point. Convective velocity on the tangent plane.}

During the quasi-static loading the contact surfaces are moving and may change. This process can be observed in the local coordinate system of the surface as a motion of a slave point $S$, defined in eqn. (26). As mentioned before for the quasi-static problems, all parameters are time dependent, where time is seen as a load parameter. Thus the "master" surface is moving and the surface vector $\boldsymbol{\rho}\left(t, \xi^{1}, \xi^{2}\right)$ as well as the normal $\mathbf{n}\left(t, \xi^{1}, \xi^{2}\right)$ are time dependent. Taking a full time derivative we obtain:

$$
\begin{aligned}
\frac{d}{d t} \mathbf{r}_{s}\left(t, \xi^{1}, \xi^{2}, \xi^{3}\right)=\frac{d}{d t} \boldsymbol{\rho} & +\frac{d}{d t}\left(\mathbf{n} \xi^{3}\right)= \\
& =\frac{\partial \boldsymbol{\rho}}{\partial t}+\frac{\partial \boldsymbol{\rho}}{\partial \xi^{j}} \dot{\xi}^{j}+\frac{\partial \mathbf{n}}{\partial t} \xi^{3}+\mathbf{n} \dot{\xi}^{3}+\frac{\partial \mathbf{n}}{\partial \xi^{j}} \dot{\xi}^{j} .
\end{aligned}
$$

With the translation velocity of the penetration point $C$ as $\mathbf{v}=\frac{\partial \boldsymbol{\rho}}{\partial t}$ and the velocity of the slave point as $\mathbf{v}_{s}=\frac{d}{d t} \mathbf{r}_{s}\left(t, \xi^{1}, \xi^{2}, \xi^{3}\right)$, the latter can be written using the Weingarten formula

$$
\mathbf{v}_{s}=\mathbf{v}+\xi^{3} \frac{\partial \mathbf{n}}{\partial t}+\mathbf{n} \dot{\xi}^{3}+\left(\boldsymbol{\rho}_{j}-\xi^{3} h_{j}^{i} \boldsymbol{\rho}_{i}\right) \dot{\xi}^{j}
$$


The convective velocities $\dot{\xi}^{i}$ and the rate of penetration $\dot{g}=\dot{\xi}^{3}$ are obtained from eqn. (37) as a projection in the local coordinate system by evaluating the dot product with the base vectors defined in eqn. (28). The vector $\frac{\partial \mathbf{n}}{\partial t}$ is orthogonal to $\mathbf{n}$ due to the fact that $\mathbf{n}$ is a unit vector:

$$
\mathbf{n} \cdot \mathbf{n}=1 \longrightarrow \frac{\partial \mathbf{n}}{\partial t} \cdot \mathbf{n}=0
$$

Evaluating then the dot product of eqn. (37) with $\mathbf{r}_{3}=\mathbf{n}$, and using the last expression (38), we obtain the projection of the relative velocity on the normal, or the full time derivative of the penetration:

$$
\dot{\xi}^{3}=\dot{g}=\left(\mathbf{v}_{s}-\mathbf{v}\right) \cdot \mathbf{n} .
$$

A dot product of eqn. (37) with the base vectors $\mathbf{r}_{i}$ gives the following expression:

$$
\left(\mathbf{v}_{s}-\mathbf{v}\right) \cdot\left(\boldsymbol{\rho}_{i}-\xi^{3} h_{i}^{k} \boldsymbol{\rho}_{k}\right)=\xi^{3} \frac{\partial \mathbf{n}}{\partial t} \cdot\left(\boldsymbol{\rho}_{i}-\xi^{3} h_{i}^{k} \boldsymbol{\rho}_{k}\right)+\left(a_{i j}-2 \xi^{3} h_{i j}+\left(\xi^{3}\right)^{2} h_{i}^{k} h_{j k}\right) \dot{\xi}^{j},
$$

from which an expression for the first two convective velocities is obtained:

$$
\dot{\xi}^{j}=\hat{a}^{i j}\left[\left(\mathbf{v}_{s}-\mathbf{v}\right) \cdot \boldsymbol{\rho}_{i}-\xi^{3}\left(\frac{\partial \mathbf{n}}{\partial t} \cdot \boldsymbol{\rho}_{i}+h_{i}^{k}\left(\mathbf{v}_{s}-\mathbf{v}\right) \cdot \boldsymbol{\rho}_{k}\right)\right],
$$

where $\hat{a}^{i j}$ are components of the inverse matrix $\left(a_{i j}-2 \xi^{3} h_{i j}+\left(\xi^{3}\right)^{2} h_{i}^{k} h_{j k}\right)$. Having taken $\xi^{3}=0$, we obtain the values of the convective velocities (41) on the tangent plane as

$$
\dot{\xi}^{j}=a^{i j}\left(\mathbf{v}_{s}-\mathbf{v}\right) \cdot \boldsymbol{\rho}_{i} .
$$

Again the assumption of a small value of the penetration $g$ allows to consider each characteristics on the tangent plane. This is a main feature of the velocity description which leads to simplification of the tangent matrix and an efficient application to non-frictional problems, see Konyukhov and Schweizerhof [10].

\subsection{Geometrical interpretation of covariant derivative and numerical realization}

The covariant derivatives require $C^{1}$ continuity of the surface. Lack of the surface continuity leads to oscillations in the characteristics, e.g. at the crossing of element boundaries. Therefore, various approaches based on the usage of a $C^{1}$ approximation of the surface with Hermite splines, NURBS etc. were developed e.g. in the following articles [30], [16], [4], [1], [22], [26]. Wriggers et. al. [30] shown that for $C^{1}$ continuous contact surfaces a continuity problem of internal parameters on the element boundary arises and proposed an algorithm for the 2D case, based on the usage of the path length of the projection point. Puso and Laursen [22] proposed to determine increments of convective coordinates in the geometric form for the 3D case. Here we construct a numerical algorithm based on a geometrical interpretation of the covariant derivative as a parallel translation, see Marsden and Hughes [18]. The result of this section will be used for the computation of the contact tractions within the return-mapping algorithm. 


\subsubsection{Continuous numerical integration algorithm for a relative motion vector $\Delta \rho$}

Consider a relative motion of the projection point $C$ on the master surface. The relative velocity vector of this motion is laying in the tangent plane, i.e.

$$
\mathbf{v}_{r}=\dot{\xi}^{i} \boldsymbol{\rho}_{i} .
$$

We are interested in the relative distance $\Delta \boldsymbol{\rho}$ which was passed by point $C$ from step $(n)$ to step $(n+1)$. For the $C^{1}$-continuous surface and continuous convective coordinates we can write the following

$$
\boldsymbol{\rho}^{(n+1)}-\boldsymbol{\rho}^{(n)}=\boldsymbol{\rho}\left(\xi^{i}+\Delta \xi^{i}\right)-\boldsymbol{\rho}\left(\xi^{i}\right)=\boldsymbol{\rho}_{i} \Delta \xi^{i}+\mathcal{O}\left(\left(\Delta \xi^{i}\right)^{2}\right)
$$

We define the incremental vector $\Delta \boldsymbol{\rho}$ at step $(n+1)$ as

$$
\Delta \boldsymbol{\rho}=\boldsymbol{\rho}_{i}^{(n+1)} \Delta \xi^{i},
$$

from which the incremental components $\Delta \xi^{i}$ are derived as

$$
\Delta \xi^{i}=\left(\Delta \boldsymbol{\rho} \cdot \boldsymbol{\rho}_{j}\right) a_{(n+1)}^{i j} .
$$

If the convective coordinates are no longer continuous then the incremental vector $\Delta \boldsymbol{\rho}$ can not be derived via eqn. (45), but it can be derived directly in the $3 \mathrm{D}$ space. For illustration, see Fig. 2, we consider at step $(n)$ two adjacent patches $A^{(n)} B^{(n)} D^{(n)} G^{(n)}$ and $G^{(n)} D^{(n)} E^{(n)} F^{(n)}$ for a $C^{1}$-continuous surface, i.e. a surface normal $\mathbf{n}$ being continuous while crossing the line $D^{(n)} G^{(n)}$, but with independently defined convective coordinates of the patches. Let $S^{(n)}$ be a slave point and $C^{(n)}$ its projection onto the patch $A^{(n)} B^{(n)} D^{(n)} G^{(n)}$ at step $(n)$. A pair of points $S^{(n)}$ and $C^{(n)}$ defines then a spatial coordinate system, eqn. (26). Now we consider a case, when at the next step $(n+1)$ the same pair is shifted into a position $S^{(n+1)}$ and $C^{(n+1)}$ with the slave point projected onto the adjacent patch $G^{(n+1)} D^{(n+1)} E^{(n+1)} F^{(n+1)}$ to obtain $C^{(n+1)}$. On the surface it can be interpreted as a motion of the projection point from position $\tilde{C}^{(n)}$ to position $C^{(n+1)}$, where the projection point has been crossing the line $D^{(n+1)} G^{(n+1)}$ (see a vector $\Delta \boldsymbol{\rho}=\tilde{\mathbf{C}}^{(n)} \mathbf{C}^{(n+1)}$ in Fig. 2). Since a moving surface is considered, point $C^{(n)}$ is shifted in the $3 \mathrm{D}$ space to the position $\tilde{C}^{(n)}$ by the vector $\mathbf{u}$. Thus, the increment vector $\Delta \rho$ is obtained in the global reference Cartesian system as

$$
\Delta \boldsymbol{\rho}=\left.\boldsymbol{\rho}_{C^{(n+1)}}\right|_{\xi_{(n+1)}^{1}}, \xi_{(n+1)}^{2}-\left.\left(\boldsymbol{\rho}_{C^{(n)}}+\mathbf{u}_{C^{(n)}}\right)\right|_{\xi_{(n)}^{1}}, \xi_{(n)}^{2} .
$$

The computation in the global reference Cartesian system clearly defines the increment vector and, therefore, allows to avoid jumps which would occur with the local convective coordinates $\xi^{i}$. It should be noted that vectors $\boldsymbol{\rho}_{C^{(n)}}$ and $\boldsymbol{\rho}_{C^{(n+1)}}$ are defined after the closest point projection procedure, therefore the information about internal variables $\xi_{1}^{(n)}, \xi_{2}^{(n)}$ must be stored. However, within the "segment-to-analytical surface"-approach the value of penetration is computed at the same integration points, i.e. $\boldsymbol{\rho}_{C^{(n)}} \equiv \boldsymbol{\rho}_{C^{(n+1)}}$. Then it is only necessary to keep the information about the increment vector $\mathbf{u}$ from the last load step in the global coordinate system. Eqn. (46) is then reduced to

$$
\Delta \xi^{i}=-\left(\mathbf{u} \cdot \boldsymbol{\rho}_{j}\right) a_{(n+1)}^{i j} .
$$

Summarizing the result we obtain the rule for the continuous numerical algorithm to compute the increment vector:

The increment vector $\Delta \boldsymbol{\rho}$ is defined in the spatial coordinate system at step $(n+1)$ by its projection in eqn. (45), where the increments $\Delta \xi^{i}$ are computed via eqn. (46) and (47), or in the case of the "segment-toanalytical surface"-approach via (48). 


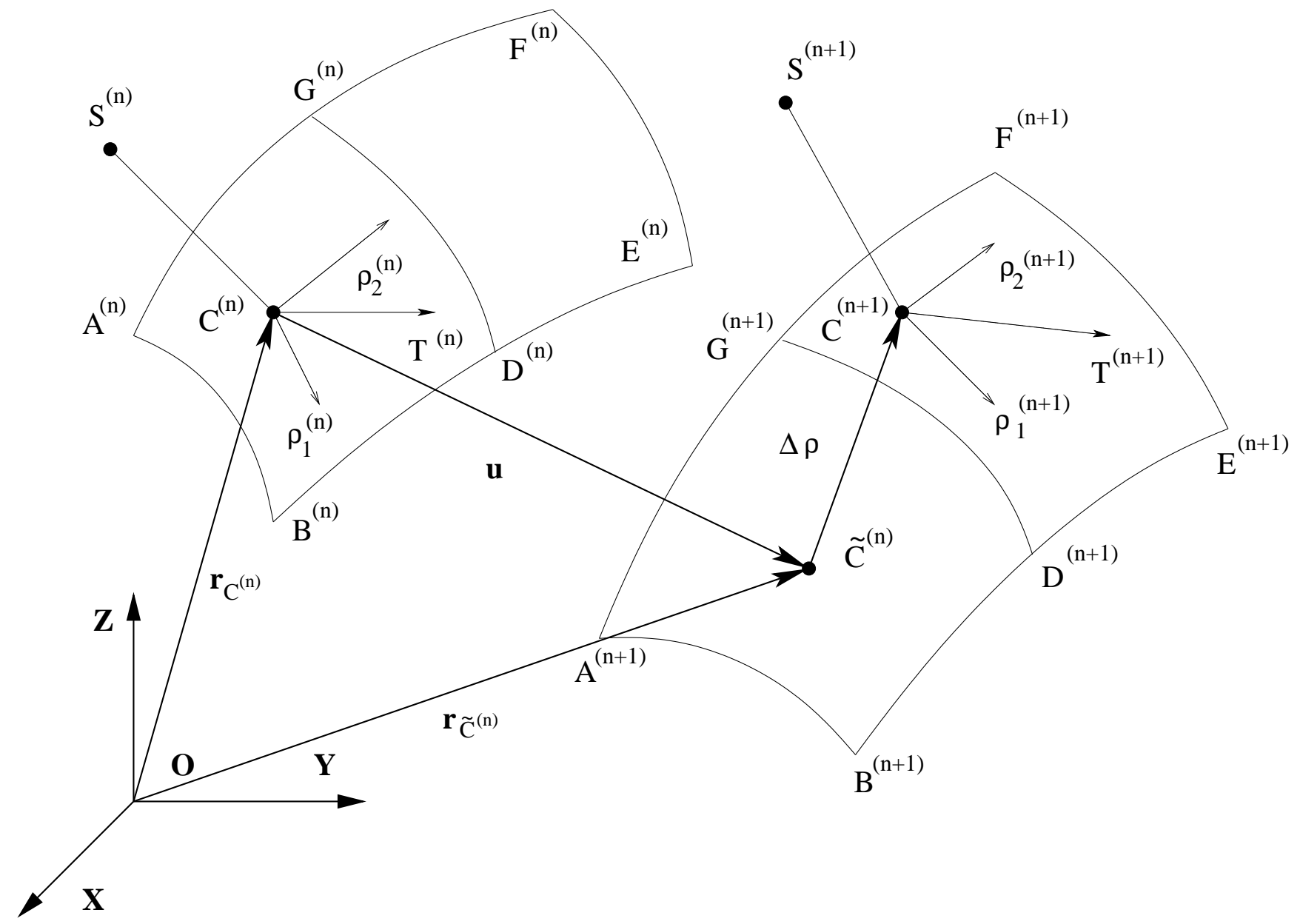

Figure 2: Contact point moving across element boundaries. Covariant derivatives. Sketch of integration scheme.

\subsubsection{Parallel translation of a vector $\mathrm{T}$ on the tangent plane.}

The full time derivative of a vector $\mathbf{T}$ in the covariant form in eqn. (35) describes its change along the tangent plane. The geometrical interpretation of the numerical increment analogy is to consider the evolution of the vector $\mathbf{T}$ by enforcing its position on the tangent plane. This operation is called "parallel translation" in differential geometry terminology, see e.g. Gray [5], Schoen [23] and application in mechanics in Marsden and Hughes [18]. This interpretation also allows to overcome a variation in the representation of the vector $\mathbf{T}$ due to different local element coordinate systems.

If $\mathbf{T}^{(n)}$ is defined at the step $(n)$, see Fig. 2 , and $\mathbf{e}_{K}$ are basis vectors of the global Cartesian coordinate system, then the vector $\mathbf{T}$ can be written in both local and global coordinate systems as

$$
\mathbf{T}=T_{i}^{(n)} a_{(n)}^{i j} \boldsymbol{\rho}_{j}^{(n)}=T_{i}^{(n)} a_{(n)}^{i j} \frac{\partial x_{(n)}^{K}}{\partial \xi^{j}} \mathbf{e}_{K} .
$$

Projections of this vector to the new basis at state $(n+1)$ gives us the vector $\mathbf{T}^{(n+1)}$ translated in parallel. This operation in the Cartesian coordinate system leads to:

$$
T_{l}^{(n+1)}=\mathbf{T}^{(n)} \cdot \boldsymbol{\rho}_{l}^{(n+1)}=T_{i}^{(n)} a_{(n)}^{i j} \frac{\partial x_{(n)}^{K}}{\partial \xi^{j}} \mathbf{e}_{K} \cdot \frac{\partial x_{(n+1)}^{M}}{\partial \xi^{l}} \mathbf{e}_{M}=T_{i}^{(n)} a_{(n)}^{i j} \frac{\partial x_{(n)}^{K}}{\partial \xi^{j}} \frac{\partial x_{(n+1)}^{K}}{\partial \xi^{l}},
$$


or in compact form

$$
T_{l}^{(n+1)}=T_{i}^{(n)} a_{(n)}^{i j}\left(\boldsymbol{\rho}_{j}^{(n)} \cdot \boldsymbol{\rho}_{l}^{(n+1)}\right) .
$$

In other words, this operation can be seen as a pull-back from the current configuration at time $(n)$ into the reference configuration and then a push-forward into the current configuration at time $(n+1)$. This procedure allows to keep continuity due to the use of the same reference configuration.

Remark. In the case of translation in a plane, the metric tensor is constant and eqn. (51) defines a standard parallel shifting

$$
T_{l}^{(n+1)}=T_{i}^{(n)} a_{(n)}^{i j}\left(\boldsymbol{\rho}_{j}^{(n)} \cdot \boldsymbol{\rho}_{l}^{(n+1)}\right)=T_{i}^{(n)} a^{i j} a_{j l}=T_{i}^{(n)} \delta_{l}^{i}=T_{l}^{(n)} .
$$

\section{Weak form for finite element formulation and regularized contact conditions}

The previous parts give us all the necessary operations to build a weak formulation. Due to the apriori small value of the penetration the weak form in the spatial coordinate system is considered on the tangent plane. A penalty method for a simple Coloumb friction law is now used as a regularization within the contact algorithm.

\subsection{Weak formulation in the spatial coordinate system.}

Now we consider the contact tractions $\mathbf{T}_{1}$ and $\mathbf{T}_{2}$ on both contact surfaces $s_{1}$ and $s_{2}$ in the current configuration. Let $\delta \mathbf{u}_{i}$ be a variation of the displacement field on the surface $s_{i}$. Then the work of the contact forces is determined in the following integral

$$
\delta W_{c}=\int_{s_{1}} \mathbf{T}_{1} \cdot \delta \mathbf{u}_{1} d s_{1}+\int_{s_{2}} \mathbf{T}_{2} \cdot \delta \mathbf{u}_{2} d s_{2},
$$

which must be added to the global work of the internal and external forces. Due to equilibrium at the contact boundary $\mathbf{T}_{1} d s_{1}=-\mathbf{T}_{2} d s_{2}$, equation (53) can be also written as

$$
\delta W_{c}=\int_{s_{1}} \mathbf{T}_{1} \cdot\left(\delta \mathbf{u}_{1}-\delta \mathbf{u}_{2}\right) d s_{1} .
$$

The integral in (54) is considered in the local coordinate system, therefore, since this point one surface must be specified as master surface and the other as slave surface. With $s_{1}$ as slave surface, the previous notation is now slightly redefined:

$\mathbf{u}_{1}=\mathbf{r}_{s}$ is a slave point; $\mathbf{u}_{2}=\boldsymbol{\rho}$ is a projection of the slave point onto the master surface;

the traction vector in the local coordinate system becomes then:

$$
\mathbf{T}_{1}=\mathbf{T}=N \mathbf{n}+T_{i} \boldsymbol{\rho}^{i}
$$

Here the traction vector is defined as a covariant vector. The variation of $\left(\mathbf{u}_{1}-\mathbf{u}_{2}\right)$ is directly obtained from the kinematic equation (37):

$$
\delta \mathbf{r}_{s}-\delta \boldsymbol{\rho}=\left(\boldsymbol{\rho}_{j}-\xi^{3} h_{j}^{i} \boldsymbol{\rho}_{i}\right) \delta \xi^{j}+\mathbf{n} \delta \xi^{3}+\xi^{3} \delta \mathbf{n} .
$$

It should be mentioned, that the variations themselves are time independent. Now the contact integral (54) can be written as:

$$
\delta W_{c}=\int_{s} N \delta \xi^{3} d s+\int_{s}\left[T_{i} \delta \xi^{i}+\xi^{3} T_{i}\left(\delta \mathbf{n} \cdot \boldsymbol{\rho}^{i}-h_{j}^{i} \delta \xi^{j}\right)\right] d s .
$$


The full integral must be considered with the variation of the convective coordinates which are obtained from eqn. (39) for the penetration as the third coordinate $g=\xi^{3}$ in the form

$$
\delta \xi^{3}=\delta g=\left(\delta \mathbf{r}_{s}-\delta \boldsymbol{\rho}\right) \cdot \mathbf{n},
$$

and from eqn. (41) for the convective coordinate $\xi^{j}$ in the form

$$
\delta \xi^{j}=\hat{a}^{i j}\left[\left(\delta \mathbf{r}_{s}-\delta \boldsymbol{\rho}\right) \cdot \boldsymbol{\rho}_{i}-\xi^{3}\left(\delta \mathbf{n} \cdot \boldsymbol{\rho}_{i}+h_{i}^{k}\left(\delta \mathbf{r}_{s}-\delta \boldsymbol{\rho}\right) \cdot \boldsymbol{\rho}_{k}\right)\right] .
$$

The full formulation with eqns. (57), (58) and (59) in the local coordinate system is very cumbersome. However, as the value of penetration $g$ must be small during the solution, which is an important feature of the current covariant description, we consider the full contact integral only on the tangent plane, i.e. $\xi^{3}=0$. Thus, we obtain the following form:

$$
\begin{aligned}
\delta W_{c}=\int_{s} N \delta g d s+ & \int_{s} T_{j} \delta \xi^{j} d s= \\
& =\int_{s} N\left(\delta \mathbf{r}_{s}-\delta \boldsymbol{\rho}\right) \cdot \mathbf{n} d s+\int_{s} T_{j} a^{i j}\left(\delta \mathbf{r}_{s}-\delta \boldsymbol{\rho}\right) \cdot \boldsymbol{\rho}_{i} d s,
\end{aligned}
$$

which is accompanied with the variation of the convective coordinates on the tangent plane in the form:

$$
\delta \xi^{j}=a^{i j}\left(\delta \mathbf{r}_{s}-\delta \boldsymbol{\rho}\right) \cdot \boldsymbol{\rho}_{i} .
$$

The formulation of the contact integral in the form presented in (60) is mostly used in contact mechanics (see Wriggers [31] and Laursen [17]).

\subsection{Regularization by the penalty method}

The contact tractions $N$ and $T_{j}$ are additional unknowns in the contact integral (60). If they are treated as independent variables, the Lagrangian multiplier method is used. If they are treated as dependent variables, additional assumptions are necessary to define the contact tractions, leading to regularization schemes. Here we follow the regularization technique as described e.g. in Kikuchi [9], Wriggers [31], [29], Laursen [17] and Zhong [34]. This regularization is based on an elasto-plastic analogy to model the Coulomb friction. Other types of regularization based on elasto-visco-plastic models of the Maxwell type and the Kelvin type are considered in Araki and Hjelmstad [2]. Besides the penalty regularization a special technique based on quadratic programming method can be applied to solve the contact problem, see e.g. Zhang et. al. [32], [33].

\subsubsection{Normal contact conditions.}

We describe contact conditions in terms of the spatial coordinate system. For normal contact they can be formulated as the Kuhn-Tucker complementary conditions for the variational problem.

1. Contact occurs when a slave point penetrates into the tangent plane: $\xi^{3}=g \leq 0$.

2. At the penetration point the normal nonnegative traction appears: $N \geq 0$.

3. The contact traction $N$ exists only, if the slave point is on the tangent plane, i.e. when $\xi^{3}=g=0: N \cdot g=0$.

The penalty method, allowing a small penetration, is often used to overcome numerical difficulties in satisfying conditions 1-3. These three conditions can be accomplished by the following regularization:

$$
N=\epsilon_{N}\langle g\rangle,
$$


where $\epsilon_{N}$ is a penalty parameter and \langle\rangle are Macauley brackets in the form

$$
\langle g\rangle=\left\{\begin{array}{lll}
0, & \text { if } & g>0 \\
g, & \text { if } & g \leq 0
\end{array} .\right.
$$

\subsubsection{Tangential contact conditions. Evolution equations.}

Additional constitutive equations are necessary for the tangential contact tractions $T_{j}$. Frictional problems in the finite element formulation are considered as quasi-static ones with the loading from zero up to a certain value. This kinematical approach allows to describe stick and slide conditions in our spatial coordinate system, see Fig. 1.

a) The slave point $S$ sticks, if its projection point $C$ is not moving on the tangent plane, i.e. has zero relative velocity $\mathbf{v}_{r}=0$.

b) The slave point $S$ slides, if during quasi-static loading there is a relative motion of its projection point $C$, i.e. $\mathbf{v}_{r} \neq 0$.

These conditions for the simplest case as a model of Coulomb dry friction can be specified as follows:

1. The slave point sticks as long as the Coulomb dry friction inequality holds

$$
\mathbf{v}_{r}=0 \quad \text { if } \quad \Phi:=\|\mathbf{T}\|-\mu N \leq 0
$$

where $\mu$ is a friction coefficient, and $\|\mathbf{T}\|$ is the absolute value of the tangential traction $\mathbf{T}$, which is computed as

$$
\|\mathbf{T}\|=\sqrt{T_{i} T_{j} a^{i j}} .
$$

2. Beyond the threshold defined by the friction condition (64) the slave point starts to slide in the direction of the relative velocity vector; the tangential tractions are then acting in the opposite direction.

$$
\text { if } \Phi>0 \quad \text { then } \exists \zeta>0 \quad \mathbf{v}_{r}=-\zeta \frac{\mathbf{T}}{\|\mathbf{T}\|},
$$

where $\zeta$ is a consistency parameter.

3. Sliding happens only if $\Phi=0$, thus

$$
\zeta \Phi=0 .
$$

Again the contact conditions lead to a lack of differentiability and, therefore, numerical problems. In order to overcome this Kikuchi [9] considered a penalty regularization for the contact functional assuming a small tangential motion in the case of sticking; a penalty regularization based on the elasto-plastic analogy was developed then in Wriggers et. al. [28], Laursen and Simo [24]. In the last article the following regularization was proposed in convective coordinates for the trial tractions:

$$
a_{i j} \dot{\xi}^{j}-\zeta \frac{T_{i}}{\|\mathbf{T}\|}=-\frac{1}{\epsilon_{T}} \frac{\partial T_{i}}{\partial t},
$$

where $\epsilon_{T}$ is a penalty parameter. Then a return-mapping algorithm known from plasticity can be used to satisfy the stick-slide condition.

From a mathematical point of view (see Marsden and Hughes [18]), it appears to be more correct to consider a parallel translation of the vector field $T_{i}\left(\xi^{i}(t)\right)$ on the master surface. In this situation the relative velocity vector $\mathbf{v}_{r}$ of the projection point $C$ on the master surface, 
see eqn. (43) must be equal to the full time derivative in the covariant form (18) of the vector $\mathbf{T}$ defined on the tangent plane in the spatial coordinate system. Thus, for the corresponding regularization we propose the following form

$$
\mathbf{v}_{r}-\zeta \frac{\mathbf{T}}{\|\mathbf{T}\|}=-\frac{1}{\epsilon_{T}} \frac{d \mathbf{T}}{d t}
$$

or employing the covariant derivative of $\mathbf{T}$ on the tangent plane in eqn. (35), we obtain the following expression for the components

$$
a_{i j} \dot{\xi}^{j}-\zeta \frac{T_{i}}{\|\mathbf{T}\|}=-\frac{1}{\epsilon_{T}}\left(\frac{\partial T_{i}}{\partial t}+\left(\frac{\partial T_{i}}{\partial \xi^{j}}-\Gamma_{i j}^{k} T_{k}\right) \dot{\xi}^{j}+h_{i}^{k} T_{k} \dot{\xi}^{3}\right)
$$

or finally, having denoted the time derivative of $T_{i}$ as

$$
\frac{d T_{i}}{d t}=\frac{\partial T_{i}}{\partial t}+\frac{\partial T_{i}}{\partial \xi^{j}} \dot{\xi}^{j}
$$

we obtain

$$
a_{i j} \dot{\xi}^{j}-\zeta \frac{T_{i}}{\|\mathbf{T}\|}=-\frac{1}{\epsilon_{T}}\left(\frac{d T_{i}}{d t}-\Gamma_{i j}^{k} T_{k} \dot{\xi}^{j}+h_{i}^{k} T_{k} \dot{\xi}^{3}\right) .
$$

In order to integrate the differential equation (72) we employ a return-mapping algorithm based on the backward Euler implicit scheme for the ordinary differential equations, see e.g. Simo and Hughes [25]. The trial step is assumed to be with sticking, therefore $\zeta=0$. The consistent backward Euler scheme for eq. (72) has the following form

$$
\begin{gathered}
\left(\delta_{k}^{i}-\left.\Gamma_{i j}^{k}\right|_{(n+1)} \xi_{(n+1)}^{j}+\left.h_{i}^{k}\right|_{(n+1)} \xi_{(n+1)}^{3}\right)\left(T^{\text {trial }}\right)_{k}^{(n+1)}= \\
=\left(T^{\text {trial }}\right)_{i}^{(n)}-\epsilon_{T}\left(a_{i j}^{(n+1)} \xi_{(n+1)}^{j}-a_{i j}^{(n)} \xi_{(n)}^{j}\right)-\left.\Gamma_{i j}^{k}\right|_{(n)} T_{k}^{(n)} \xi_{(n)}^{j}+\left.h_{i}^{k}\right|_{(n)} \xi_{(n)}^{3} T_{k}^{(n)},
\end{gathered}
$$

which can be seen as a backward scheme for the following ordinary differential equations

$$
\frac{d T_{i}}{d t}=\left(-\epsilon_{T} a_{i j}+\Gamma_{i j}^{k} T_{k}\right) \dot{\xi}^{j}-h_{i}^{k} T_{k} \dot{\xi}^{3} .
$$

The system of ordinary differential equations for the computation of the tangential traction (74) is called the evolution equations. They are important for the linearization process. Keeping the form with the covariant derivatives (74) instead of the form in eqn. (68) leads to a symmetrical tangent matrix for sticking, while as used in Laursen and Simo [15], [14], the form (68) leads to a non-symmetrical tangent matrix for the arbitrary 3D case.

Remark 1. Consider the backward Euler scheme (73) in the case with a linear approximation of the master surface. Then, having taken all Christoffel symbols and components of the curvature tensor as zero, we obtain the following equations:

$$
\left(T^{t r}\right)_{i}^{(n+1)}=T_{i}^{(n)}-\epsilon_{T} a_{i j}\left(\xi_{(n+1)}^{j}-\xi_{(n)}^{j}\right) .
$$

This algorithm can be found in Laursen [17] for the trial step solution of equation (68).

Any analysis based on equation (73) becomes computationally rather expensive, because a full matrix appears on the left side and additional history variables $\left.\Gamma_{i j}^{k}\right|_{(n)}, a_{i j}^{(n)}$ have to be used. Moreover, the integration scheme (73) as well as (75) suffers from jumps occurring at element boundaries due to the different internal coordinates $\xi^{i}$. Thus, we propose a discrete analog of the evolution equations (74) for the numerical computation

$$
\Delta \mathbf{T}=-\epsilon_{T} \Delta \boldsymbol{\rho} .
$$

The application of the results of section 2.3 to eqn. (76) together with the sliding condition leads to the following return-mapping scheme: 
Trial step.

$$
\left.\begin{array}{l}
N^{(n+1)}=\epsilon_{N}\left\langle g^{(n+1)}\right\rangle \\
\left(T^{t r}\right)_{i}^{(n+1)}=T_{k}^{(n)} a_{(n)}^{k j}\left(\boldsymbol{\rho}_{j}^{(n)} \cdot \boldsymbol{\rho}_{i}^{(n+1)}\right)-\epsilon_{T} \Delta \xi^{j} a_{i j}^{(n+1)} \\
\Phi_{(n+1)}^{t r}:=\left\|\mathbf{T}_{(n+1)}^{t r}\right\|-\mu N^{(n+1)} \\
\left\|\mathbf{T}_{(n+1)}^{t r}\right\|=\sqrt{\left(T^{t r}\right)_{i}^{(n+1)}\left(T^{t r}\right)_{j}^{(n+1)} a_{(n+1)}^{i j}}
\end{array}\right\},
$$

where $\Delta \xi^{j}$ is obtained as

$$
\Delta \xi^{j}=\left\{\begin{array}{cc}
\left(\Delta \boldsymbol{\rho} \cdot \boldsymbol{\rho}_{k}\right) a_{(n+1)}^{j k} & \begin{array}{l}
\text { for node-to-surface (NTS) and } \\
\text { surface-to-surface (STS) approaches, where }
\end{array} \\
\qquad \boldsymbol{\rho}=\left.\boldsymbol{\rho}_{C^{(n+1)}}\right|_{\xi_{(n+1)}^{1}}, \xi_{(n+1)}^{2}-\left.\left(\boldsymbol{\rho}_{C^{(n)}}+\mathbf{u}_{C^{(n)}}\right)\right|_{\xi_{(n)}^{1}}, \xi_{(n)}^{2} \\
-\left(\mathbf{u} \cdot \boldsymbol{\rho}_{k}\right) a_{(n+1)}^{k j} & \text { for segment-to-analytical surface (STAS) approach }
\end{array} .\right.
$$

Return mapping. The stick-slip condition is checked within the return mapping process:

$$
T_{i}^{(n+1)}=\left\{\begin{array}{c}
\left(T^{t r}\right)_{i}^{(n+1)} \text { if } \Phi_{(n+1)}^{t r} \leq 0 \quad(\text { stick }) \\
\mu N^{(n+1) \frac{\left(T^{t r}\right)_{i}^{(n+1)}}{\left\|\mathbf{T}_{(n+1)}^{t r}\right\|}} \text { if } \Phi_{(n+1)}^{t r}>0 \quad \text { (slide) }
\end{array} .\right.
$$

Remark 2. The regularized frictional problem is strictly path-dependent: it follows from the fact that the contact tractions $T_{i}$ in the contact functional in eqn. (60) must satisfy the evolution equations (74). The return-mapping algorithm for the incremental solution, as is known, is unconditionally stable, but a problem of choosing the displacement increments arises due to the correct definition of sticking and sliding zones. A simple a-priori estimation will be proposed further for the numerical example.

Remark 3. For 2D problems Krstulovic-Opara and Wriggers [12] proposed the so-called moving cone description. Under the assumption of Remark 1, now a point of the cone axis on the tangent plane with coordinates $\xi_{0}^{1}, \xi_{0}^{2}$ is considered. One can show that the friction condition (77. 3) together with eqn. (75) defines an ellipse on the tangent plane, which can be obtained by projection of the frictional cone onto the tangent plane. For a stick case the initial frictional forces $T_{i}$ are zero at the initial point $\xi_{(0)}^{1}, \xi_{(0)}^{2}$ in algorithm (75). Considering the absolute value $\|\mathbf{T}\|$ in eqn. (65) at step $(n)$ we obtain

$$
\left\|\mathbf{T}^{(n)}\right\|^{2}=T_{i}^{(n)} T_{j}^{(n)} a^{i j}=\epsilon_{T} a_{i k}\left(\xi_{(n)}^{k}-\xi_{(0)}^{k}\right) \epsilon_{T} a_{j l}\left(\xi_{(n)}^{l}-\xi_{(0)}^{l}\right) a^{i j}=\epsilon_{T}^{2} a_{k l}\left(\xi_{(n)}^{k}-\xi_{(0)}^{k}\right)\left(\xi_{(n)}^{l}-\xi_{(0)}^{l}\right) .(80
$$

Having taken an incremental analog of the differential of length in eqn. (9) together with eqn. (77. 3), we can find that

$$
\Delta l^{2}=\epsilon_{T}^{2} a_{k l}\left(\xi_{(n)}^{k}-\xi_{(0)}^{k}\right)\left(\xi_{(n)}^{l}-\xi_{(0)}^{l}\right) \leq(\mu N)^{2} .
$$

Eqn. (81) defines an ellipse as allowable domain inside which the projection point $C$ can move in the case of sticking leading to a symmetric tangent matrix finally. 


\section{Consistent linearization}

The idea behind the consistent linearization for a Newton type solution process is to exploit the full material time derivative in the form of the covariant derivative in the spatial coordinate system, see sections 2.1.2 and 2.2.2, together with the evolution equations for the contact tangent frictional forces (74).

\subsection{Linearization of the normal contact expression}

The contact integral, see e.g. eqn. (54) is computed over the "slave" surface, which is defined by a set of "slave" points. Each parameter in the contact integral is considered in the spatial local coordinate system of the "master" surface, (i.e. as a function of the convective coordinates $\xi^{i}$ ), therefore, linearization of the "slave" surface element $d s$ will not be included in process. Thus $d s$ is assumed to remain constant within linearization. Further it must be noted that the use of different quadrature schemes for the computation of the contact integral may lead to different contact elements.

The normal part of the contact integral (60) has the following form:

$$
\delta W_{c}^{N}=\int_{s} \epsilon_{N}\langle g\rangle \delta g d s=\int_{s} \epsilon_{N}\left\langle\left(\mathbf{r}_{s}-\boldsymbol{\rho}\right) \cdot \mathbf{n}\right\rangle\left(\delta \mathbf{r}_{s}-\delta \boldsymbol{\rho}\right) \cdot \mathbf{n} d s .
$$

The details of the linearization of the normal part $\delta W_{c}^{N}$ and the application to the non-frictional problems are outlined in Konyukhov and Schweizerhof [10]. Here we only include the result for the full normal tangent matrix:

$$
\begin{gathered}
D\left(\delta W_{c}^{N}\right)= \\
=\int_{S} \epsilon_{N} H(-g)\left(\delta \mathbf{r}_{s}-\delta \boldsymbol{\rho}\right) \cdot(\mathbf{n} \otimes \mathbf{n})\left(\mathbf{v}_{s}-\mathbf{v}\right) d S- \\
-\int_{S} \epsilon_{N} H(-g) g\left(\delta \boldsymbol{\rho}_{, j} \cdot a^{i j}\left(\mathbf{n} \otimes \boldsymbol{\rho}_{i}\right)\left(\mathbf{v}_{s}-\mathbf{v}\right)+\left(\delta \mathbf{r}_{s}-\delta \boldsymbol{\rho}\right) \cdot a^{i j}\left(\boldsymbol{\rho}_{j} \otimes \mathbf{n}\right) \mathbf{v}_{, i}\right) d S- \\
-\int_{S} \epsilon_{N} H(-g) g\left(\delta \mathbf{r}_{s}-\delta \boldsymbol{\rho}\right) \cdot h^{i j}\left(\boldsymbol{\rho}_{i} \otimes \boldsymbol{\rho}_{j}\right)\left(\mathbf{v}_{s}-\mathbf{v}\right) d S .
\end{gathered}
$$

The full contact tangent matrix is subdivided into the main part eqn. (83), the "rotational" part (83 a) and the "curvature" part $(83$ b). The last two terms are small due to the small value of the penetration $g$. The "rotational" part contains derivatives of $\delta \boldsymbol{\rho}$ and $\mathbf{v}$ with respect to the convective coordinates $\xi^{j}$ and, therefore, represents the rotation of a contact surface during the incremental solution procedure. The "curvature" part contains components of the curvature tensor $h^{i j}$ and, therefore, represents the change of the curvature of the master surface.

\subsection{Linearization of the tangential contact expression}

The tangential part of the contact integral (60)

$$
\delta W_{c}^{T}=\int_{S} T_{i} \delta \xi^{i} d s
$$

has to be considered together with the evolution equations (74) and the return mapping algorithm eqn. (77), (78), (79). The cases of sticking and sliding have to be treated separately. 
For the linearization either a covariant or a contravariant component, two operators, based on the covariant derivative are necessary. The operator for the linearization of the contravariant component has the form

$$
L\left(x^{i}\right) \equiv\left(\frac{\partial}{\partial t}+\dot{\xi}^{j} \nabla_{j}\right)\left(x^{i}\right)=\frac{\partial x^{i}}{\partial t}+\left(\frac{\partial x^{i}}{\partial \xi^{j}}+\Gamma_{k j}^{i} x^{k}\right) \dot{\xi}^{j}
$$

and the linearization operator for the covariant component has the form

$$
L\left(x_{i}\right) \equiv\left(\frac{\partial}{\partial t}+\dot{\xi}^{j} \nabla_{j}\right)\left(x_{i}\right)=\frac{\partial x_{i}}{\partial t}+\left(\frac{\partial x_{i}}{\partial \xi^{j}}-\Gamma_{i j}^{k} x_{i}\right) \dot{\xi}^{j} .
$$

It is obvious that the Christoffel symbols disappear in the final result after the linearization of the scalar, i.e. the full time derivative of the scalar is the covariant derivative of the scalar

$$
\begin{gathered}
L\left(x^{i} v_{i}\right)=\left\{\frac{\partial x^{i}}{\partial t}+\left(\frac{\partial x^{i}}{\partial \xi^{j}}+\Gamma_{k j}^{i} x^{k}\right) \dot{\xi}^{j}\right\} v_{i}+\left\{\frac{\partial v_{i}}{\partial t}+\left(\frac{\partial v_{i}}{\partial \xi^{j}}-\Gamma_{i j}^{k} v_{i}\right) \dot{\xi}^{j}\right\} x^{i}= \\
=\left\{\frac{\partial x^{i}}{\partial t}+\frac{\partial x^{i}}{\partial \xi^{j}} \dot{\xi}^{j}\right\} v_{i}+\left\{\frac{\partial v_{i}}{\partial t}+\frac{\partial v_{i}}{\partial \xi^{j}} \dot{\xi}^{j}\right\} x^{i}=v_{i} \frac{d x^{i}}{d t}+x^{i} \frac{d v_{i}}{d t} .
\end{gathered}
$$

Therefore, the linearization leads to the following expression

$$
D_{v}\left(\delta W_{c}^{T}\right)=\int_{s}\left(\delta \xi^{i} \frac{d T_{i}}{d t}+T_{i} \frac{d \delta \xi^{i}}{d t}\right) d s .
$$

As the handling of the complete expression is rather complex, we focus on each term separately in the following.

\subsubsection{Linearization of $\delta \xi^{i}$}

The linearization of the variation of the convective coordinates $\delta \xi^{i}$ is one of the important parts which requires the results about differential operations in the spatial coordinate system from section 2.2.2 together with the tensor algebra operations on the tangent plane. The full time derivative gives

$$
\left.L\left(\delta \xi^{i}\right)=\left\{\frac{\partial}{\partial t}+\frac{\partial}{\partial \xi^{j}} \dot{\xi}^{j}\right\}\left(\delta \xi^{i}\right)=\frac{d a^{i k}}{d t}\left(\delta \mathbf{r}_{s}-\delta \boldsymbol{\rho}\right) \cdot \boldsymbol{\rho}_{k}+a^{i k} \frac{d}{d t}\left[\left(\delta \mathbf{r}_{s}-\delta \boldsymbol{\rho}\right) \cdot \boldsymbol{\rho}_{k}\right)\right] .
$$

Linearization of $\left(\delta \mathbf{r}_{s}-\delta \boldsymbol{\rho}\right) \cdot \boldsymbol{\rho}_{k}$ requires the application of the Gauss-Kodazzi formula (14).

$$
\begin{aligned}
\left.\frac{d}{d t}\left[\left(\delta \mathbf{r}_{s}-\delta \boldsymbol{\rho}\right) \cdot \boldsymbol{\rho}_{k}\right)\right]= & \\
& \left.=\left(\left(\delta \mathbf{r}_{s}-\delta \boldsymbol{\rho}\right)_{, j} \cdot \boldsymbol{\rho}_{k}\right) \dot{\xi}^{j}+\left(\delta \mathbf{r}_{s}-\delta \boldsymbol{\rho}\right) \cdot \mathbf{v}_{k}\right)+ \\
+ & \Gamma_{k j}^{l}\left(\left(\delta \mathbf{r}_{s}-\delta \boldsymbol{\rho}\right) \cdot \boldsymbol{\rho}_{l}\right) \dot{\xi}^{j}+h_{k j}\left(\left(\delta \mathbf{r}_{s}-\delta \boldsymbol{\rho}\right) \cdot \mathbf{n}\right) \dot{\xi}^{j}
\end{aligned}
$$

Linearization of the contravariant components $a^{i j} \quad$ was already given in the section 2.2.2. 
Simplification of $\frac{d}{d t} \delta \xi^{i}$. The final formula is long, but can be simplified. In addition, the following transformations are cumbersome but necessary to show the symmetry of the tangent matrix in the case of sticking. Summarizing the results in one formula, we obtain

$$
\begin{gathered}
\frac{d}{d t}\left(\delta \xi^{i}\right)= \\
=-a^{i m} a^{n k}\left(\mathbf{v}_{m} \cdot \boldsymbol{\rho}_{n}\right)\left(\left(\delta \mathbf{r}_{s}-\delta \boldsymbol{\rho}\right) \cdot \boldsymbol{\rho}_{k}\right) \\
-a^{i m} a^{n k}\left(\boldsymbol{\rho}_{m} \cdot \mathbf{v}_{n}\right)\left(\left(\delta \mathbf{r}_{s}-\delta \boldsymbol{\rho}\right) \cdot \boldsymbol{\rho}_{k}\right) \\
-a^{i m} a^{n k} \Gamma_{m j}^{l}\left(\boldsymbol{\rho}_{l} \cdot \boldsymbol{\rho}_{n}\right) \dot{\xi}\left(\left(\delta \mathbf{r}_{s}-\delta \boldsymbol{\rho}\right) \cdot \boldsymbol{\rho}_{k}\right) \\
-a^{i m} a^{n k} \Gamma_{n j}^{l}\left(\boldsymbol{\rho}_{m} \cdot \boldsymbol{\rho}_{l}\right) \dot{\xi}^{j}\left(\left(\delta \mathbf{r}_{s}-\delta \boldsymbol{\rho}\right) \cdot \boldsymbol{\rho}_{k}\right) \\
+2 a^{i m} a^{n k} h_{m n} \dot{\xi}^{3}\left(\left(\delta \mathbf{r}_{s}-\delta \boldsymbol{\rho}\right) \cdot \boldsymbol{\rho}_{k}\right) \\
\left.+a^{i k}\left(\left(\delta \mathbf{r}_{s}-\delta \boldsymbol{\rho}\right)\right), \boldsymbol{\rho}_{k}\right) \dot{\xi}^{j} \\
+a^{i k}\left(\delta \mathbf{r}_{s}-\delta \boldsymbol{\rho}\right) \cdot \mathbf{v}_{k} \\
+a^{i k} \Gamma_{k j}^{l}\left(\left(\delta \mathbf{r}_{s}-\delta \boldsymbol{\rho}\right) \cdot \boldsymbol{\rho}_{l}\right) \dot{\xi}^{j} \\
+a^{i k} h_{k j}\left(\left(\delta \mathbf{r}_{s}-\delta \boldsymbol{\rho}\right) \cdot \mathbf{n}\right) \dot{\xi}^{j} .
\end{gathered}
$$

The nine parts in eqn. (91) will be tremendously simplified, if we take into account the expression for the convective velocities (42) and consider tensor operations on the tangent plane. The following five transformations will lead to a simple structure:

a.

The sum of the terms (91) and (91 f) becomes zero on the surface:

$$
-a^{i m} a^{n k}\left(\mathbf{v}_{m} \cdot \boldsymbol{\rho}_{n}\right)\left(\left(\delta \mathbf{r}_{s}-\delta \boldsymbol{\rho}\right) \cdot \boldsymbol{\rho}_{k}\right)+a^{i k}\left(\delta \mathbf{r}_{s}-\delta \boldsymbol{\rho}\right) \cdot \mathbf{v}_{k}=0 .
$$

In order to show this the dot product in the second term is expressed on the tangent plane, i.e. as double sum with the surface metric tensor components $a^{i j}$ :

$$
\begin{gathered}
a^{i m}\left(\delta \mathbf{r}_{s}-\delta \boldsymbol{\rho}\right) \cdot \mathbf{v}_{m}=a^{i m}\left(\left(\delta \mathbf{r}_{s}-\delta \boldsymbol{\rho}\right) \cdot \boldsymbol{\rho}_{k}\right) \boldsymbol{\rho}^{k} \cdot\left(\mathbf{v}_{m} \cdot \boldsymbol{\rho}_{n}\right) \boldsymbol{\rho}^{n}= \\
=a^{i m} a^{k n}\left(\left(\delta \mathbf{r}_{s}-\delta \boldsymbol{\rho}\right) \cdot \boldsymbol{\rho}_{k}\right)\left(\mathbf{v}_{m} \cdot \boldsymbol{\rho}_{n}\right),
\end{gathered}
$$

from which (92) is obtained.

b.

The sum of the terms $(91 b)$ and $(91 \mathrm{~g})$ becomes zero on the surface:

$$
\begin{aligned}
-a^{i m} a^{n k} \Gamma_{m j}^{l} & \left(\boldsymbol{\rho}_{l} \cdot \boldsymbol{\rho}_{n}\right) \dot{\xi}^{j}\left(\left(\delta \mathbf{r}_{s}-\delta \boldsymbol{\rho}\right) \cdot \boldsymbol{\rho}_{k}\right)+a^{i k} \Gamma_{k j}^{l}\left(\left(\delta \mathbf{r}_{s}-\delta \boldsymbol{\rho}\right) \cdot \boldsymbol{\rho}_{l}\right) \dot{\xi}^{j}= \\
= & \left(-a^{i m} a^{n k} a_{l n} \Gamma_{m j}^{l}\left(\left(\delta \mathbf{r}_{s}-\delta \boldsymbol{\rho}\right) \cdot \boldsymbol{\rho}_{k}\right)+a^{i k} \Gamma_{k j}^{l}\left(\left(\delta \mathbf{r}_{s}-\delta \boldsymbol{\rho}\right) \cdot \boldsymbol{\rho}_{l}\right)\right) \dot{\xi}^{j}= \\
& =\left(-a^{i m} a_{l}^{k} \Gamma_{m j}^{l}\left(\left(\delta \mathbf{r}_{s}-\delta \boldsymbol{\rho}\right) \cdot \boldsymbol{\rho}_{k}\right)+a^{i k} \Gamma_{k j}^{l}\left(\left(\delta \mathbf{r}_{s}-\delta \boldsymbol{\rho}\right) \cdot \boldsymbol{\rho}_{l}\right)\right) \dot{\xi}^{j}= \\
& =\left(-a^{i m} \Gamma_{m j}^{k}\left(\left(\delta \mathbf{r}_{s}-\delta \boldsymbol{\rho}\right) \cdot \boldsymbol{\rho}_{k}\right)+a^{i k} \Gamma_{k j}^{l}\left(\left(\delta \mathbf{r}_{s}-\delta \boldsymbol{\rho}\right) \cdot \boldsymbol{\rho}_{l}\right)\right) \dot{\xi}^{j}=0 .
\end{aligned}
$$

Here the properties of the covariant and contravariant components (4) and (5) have been used.

c.

The sum of $(91 \mathrm{a})$ and $(91 \mathrm{e})$ leads to a symmetrical rotational part. We start with using the expression for the convective velocities (42):

$$
-a^{i m} a^{n k}\left(\boldsymbol{\rho}_{m} \cdot \mathbf{v}_{n}\right)\left(\left(\delta \mathbf{r}_{s}-\delta \boldsymbol{\rho}\right) \cdot \boldsymbol{\rho}_{k}\right)+a^{i k}\left(\left(\delta \mathbf{r}_{s}-\delta \boldsymbol{\rho}\right)_{, j} \cdot \boldsymbol{\rho}_{k}\right) \dot{\xi}^{j}=
$$




$$
\begin{gathered}
=-a^{i l} a^{j k}\left(\boldsymbol{\rho}_{l} \cdot \mathbf{v}_{j}\right)\left(\left(\delta \mathbf{r}_{s}-\delta \boldsymbol{\rho}\right) \cdot \boldsymbol{\rho}_{k}\right)-a^{i k} a^{j l}\left(\delta \boldsymbol{\rho}_{, j} \cdot \boldsymbol{\rho}_{k}\right)\left(\left(\mathbf{v}_{s}-\mathbf{v}\right) \cdot \boldsymbol{\rho}_{l}\right)= \\
=-\left(\delta \mathbf{r}_{s}-\delta \boldsymbol{\rho}\right) a^{i l} a^{j k} \boldsymbol{\rho}_{k} \otimes \boldsymbol{\rho}_{l} \mathbf{v}_{j}-\delta \boldsymbol{\rho}_{, j} a^{i k} a^{j l} \boldsymbol{\rho}_{k} \otimes \boldsymbol{\rho}_{l}\left(\mathbf{v}_{s}-\mathbf{v}\right) .
\end{gathered}
$$

The final expression is found via the tensor product.

d.

After grouping $(91 d)$ with $(91 h)$, we obtain

$$
\begin{gathered}
2 a^{i m} a^{n k} h_{m n} \dot{\xi}^{3}\left(\left(\delta \mathbf{r}_{s}-\delta \boldsymbol{\rho}\right) \cdot \boldsymbol{\rho}_{k}\right)+a^{i k} h_{k j}\left(\left(\delta \mathbf{r}_{s}-\delta \boldsymbol{\rho}\right) \cdot \mathbf{n}\right) \dot{\xi}^{j}= \\
=a^{i m} a^{n k} h_{m n} \dot{\xi}^{3}\left(\left(\delta \mathbf{r}_{s}-\delta \boldsymbol{\rho}\right) \cdot \boldsymbol{\rho}_{k}\right)+ \\
\left.a^{i m} a^{n k} h_{m n}\left(\mathbf{v}_{s}-\mathbf{v}\right) \cdot \mathbf{n}\right)\left(\left(\delta \mathbf{r}_{s}-\delta \boldsymbol{\rho}\right) \cdot \boldsymbol{\rho}_{k}\right)+a^{i k} a^{j m} h_{k j}\left(\left(\delta \mathbf{r}_{s}-\delta \boldsymbol{\rho}\right) \cdot \mathbf{n}\right)\left(\left(\mathbf{v}_{s}-\mathbf{v}\right) \cdot \boldsymbol{\rho}_{m}\right)=
\end{gathered}
$$

In order to show the symmetry of the part in eqn. $(95$ a), the tensor product and contravariant components of the curvature tensor eqn. (12) are used. For a reduction of eqn. (95) the equation for the variation of the convective velocity (42) and mixed components of the curvature tensor are taken, leading finally to

$$
\begin{aligned}
& =h_{n}^{i} \dot{\xi}^{3} \delta \xi^{n}+ \\
& \quad+h^{i j}\left(\delta \mathbf{r}_{s}-\delta \boldsymbol{\rho}\right) \cdot\left(\boldsymbol{\rho}_{j} \otimes \mathbf{n}+\mathbf{n} \otimes \boldsymbol{\rho}_{j}\right)\left(\mathbf{v}_{s}-\mathbf{v}\right) .
\end{aligned}
$$

The last part (96 a) defines the curvature part of the tangent matrix.

e.

The equation for the variation of the convective velocity (42) is used to simplify $(91 \mathrm{c})$ :

$$
-a^{i m} a^{n k} \Gamma_{n j}^{l}\left(\boldsymbol{\rho}_{m} \cdot \boldsymbol{\rho}_{l}\right) \dot{\xi}^{j}\left(\left(\delta \mathbf{r}_{s}-\delta \boldsymbol{\rho}\right) \cdot \boldsymbol{\rho}_{k}\right)
$$

$$
=-\Gamma_{k j}^{i} \dot{\xi}^{j} \delta \xi^{k} .
$$

The resulting parts in eqn. (96) and (97) remain untransformed, however they will disappear in both sticking and sliding cases, after taking into account the fully linearized contact integral together with the evolution equations (74) as shown in the next section.

Summarizing the result of the complete transformation, we obtain

$$
\begin{gathered}
\frac{d}{d t}\left(\delta \xi^{i}\right)= \\
=-\left(\delta \mathbf{r}_{s}-\delta \boldsymbol{\rho}\right) a^{i l} a^{j k} \boldsymbol{\rho}_{k} \otimes \boldsymbol{\rho}_{l} \mathbf{v}_{j}-\delta \boldsymbol{\rho}_{, j} a^{i k} a^{j l} \boldsymbol{\rho}_{k} \otimes \boldsymbol{\rho}_{l}\left(\mathbf{v}_{s}-\mathbf{v}\right) \\
+h^{i j}\left(\delta \mathbf{r}_{s}-\delta \boldsymbol{\rho}\right) \cdot\left(\boldsymbol{\rho}_{j} \otimes \mathbf{n}+\mathbf{n} \otimes \boldsymbol{\rho}_{j}\right)\left(\mathbf{v}_{s}-\mathbf{v}\right)+ \\
+h_{n}^{i} \dot{\xi}^{3} \delta \xi^{n}-\Gamma_{k j}^{i} \dot{\xi}^{j} \delta \xi^{k} .
\end{gathered}
$$

Thus, the full time derivative consists of a symmetrical rotational part (98), a symmetrical pure curvature part $(98 a)$ and a connection part with the Christoffel symbols $(98$ b), describing the connection properties. 


\subsubsection{Sticking}

In the sticking case, the trial tangential traction terms $T_{i}$ are identical with the real traction, therefore, the linearized traction terms are obtained from the evolution equation in (74) directly. Starting with eqn. (88) and taking into account Remark 1 in section 3.2 together with the evolution equation (74), and eqn. (98), (98 $a)$, (98 b) we finally obtain

$$
\begin{gathered}
D_{v}\left(\delta W_{c}^{T}\right)= \\
=\int_{s}\left((-\epsilon_{T} a_{i j}+\underbrace{\left.\Gamma_{i j}^{k} T_{k}\right) \dot{\xi}^{j}-h_{i}^{k} T_{k} \dot{\xi}^{3}}) \delta \xi^{i} d s+\right. \\
+\int_{s} T_{i}\left[-\left(\left(\delta \mathbf{r}_{s}-\delta \boldsymbol{\rho}\right) a^{i l} a^{j k} \boldsymbol{\rho}_{k} \otimes \boldsymbol{\rho}_{l} \mathbf{v}_{j}+\delta \boldsymbol{\rho}_{, j} a^{i k} a^{j l} \boldsymbol{\rho}_{k} \otimes \boldsymbol{\rho}_{l}\left(\mathbf{v}_{s}-\mathbf{v}\right)\right)+\right. \\
+h^{i j}\left(\delta \mathbf{r}_{s}-\delta \boldsymbol{\rho}\right) \cdot\left(\boldsymbol{\rho}_{j} \otimes \mathbf{n}+\mathbf{n} \otimes \boldsymbol{\rho}_{j}\right)\left(\mathbf{v}_{s}-\mathbf{v}\right)+ \\
+\underbrace{h_{n}^{i} \dot{\xi}^{3} \delta \xi^{n}-\Gamma_{k j}^{i} \dot{\xi}^{j} \delta \xi^{k}}] d s .
\end{gathered}
$$

Using the tensor notation and the equation for convective velocities (42) for the main part $a_{i j} \dot{\xi}^{j} \delta \xi^{i}$, we obtain the following form for the tangential tangent matrix in the case of sticking.

$$
\begin{gathered}
D_{v}\left(\delta W_{c}^{T}\right)= \\
-\varepsilon_{T} \int_{s}\left(\delta \mathbf{r}_{s}-\delta \boldsymbol{\rho}\right) a^{i j} \boldsymbol{\rho}_{i} \otimes \boldsymbol{\rho}_{j}\left(\mathbf{v}_{s}-\mathbf{v}\right) d s \\
-\int_{s} T_{i}\left(\left(\delta \mathbf{r}_{s}-\delta \boldsymbol{\rho}\right) a^{i l} a^{j k} \boldsymbol{\rho}_{k} \otimes \boldsymbol{\rho}_{l} \mathbf{v}_{j}+\delta \boldsymbol{\rho}_{, j} a^{i k} a^{j l} \boldsymbol{\rho}_{k} \otimes \boldsymbol{\rho}_{l}\left(\mathbf{v}_{s}-\mathbf{v}\right)\right) d s \\
+\int_{s} T_{i} h^{i j}\left(\delta \mathbf{r}_{s}-\delta \boldsymbol{\rho}\right) \cdot\left(\boldsymbol{\rho}_{j} \otimes \mathbf{n}+\mathbf{n} \otimes \boldsymbol{\rho}_{j}\right)\left(\mathbf{v}_{s}-\mathbf{v}\right) d s .
\end{gathered}
$$

As we have a conservative problem for sticking it is obvious that the symmetric form is correct.

Similar to the normal tangent matrix, the tangential tangent matrix can be subdivided into a main (100), a rotational (100 a) and a pure curvature part (100 b).

Remark 1. The artificial non-symmetry of the tangent matrix for the stick condition, based on the evolution equation (68) was mentioned by Laursen and Simo in [15], [17] and [14]. As an appropriate alternative within a solution scheme, a symmetrization based on a split technique with the Augmented Lagrangian method was proposed by Laursen in [24]. Wriggers [31], suggested to use the consistent linearization of the sticking conditions in the form $\left\|\mathbf{r}_{s}-\boldsymbol{\rho}\right\|^{2}$ directly, which then leads to the correct symmetric matrix. Here, it becomes obvious, that it is particularly important to use the evolution equation in the form of the covariant derivatives (see eqn. 74) together with the linearization of the metric components $a_{i j}$ as 3D metric components $g_{i j}$ (see eqn. 30). This allows to avoid the artificial non-symmetry and obtain the correct symmetric tangent matrix for sticking. 


\subsubsection{Sliding}

The expressions for the linearized variation of the convective velocity eqn. (98), (98 $a$ ) and (98 b) are also used in this case. In addition, the tangential force in the case of sliding, see eqn. (79) of the return-mapping algorithm, has to be linearized.

$$
\frac{d T_{i}^{(n+1)}}{d t}=\frac{d}{d t}\left(\mu N^{(n+1)} \frac{\left(T^{t r}\right)_{i}^{(n+1)}}{\left\|\mathbf{T}_{(n+1)}^{t r}\right\|}\right)=\mu \frac{d N^{(n+1)}}{d t} \frac{\left(T^{t r}\right)_{i}^{(n+1)}}{\left\|\mathbf{T}_{(n+1)}^{t r}\right\|}+\mu N^{(n+1)} \frac{d}{d t}\left(\frac{\left(T^{t r}\right)_{i}^{(n+1)}}{\left\|\mathbf{T}_{(n+1)}^{t r}\right\|}\right)
$$

For the derivative of the unit vector on the tangent plane

$$
\mathbf{e}=\frac{\left(T^{\text {trial }}\right)_{i}^{(n+1)}}{\left\|\mathbf{T}_{(n+1)}^{\text {trial }}\right\|} a^{i j} \boldsymbol{\rho}_{j}
$$

we will use the following formula, see Simo and Hughes [25]

$$
\frac{d \mathbf{e}}{d \mathbf{T}}=\frac{1}{\|\mathbf{T}\|}[\mathbf{I}-\mathbf{e} \otimes \mathbf{e}]
$$

and the chain rule

$$
\frac{d \mathbf{e}}{d t}=\frac{d \mathbf{e}}{d \mathbf{T}} \frac{d \mathbf{T}}{d t}=\frac{1}{\|\mathbf{T}\|}[\mathbf{I}-\mathbf{e} \otimes \mathbf{e}] \frac{d \mathbf{T}}{d t} .
$$

Here the full time derivative of the tangential traction $\frac{d \mathbf{T}}{d t}$ is given by the evolution equation (74). The tensor operations are considered on the tangent plane:

$$
\begin{gathered}
{[\mathbf{I}-\mathbf{e} \otimes \mathbf{e}] \frac{d \mathbf{T}}{d t}=\left[a^{i j} \boldsymbol{\rho}_{i} \otimes \boldsymbol{\rho}_{j}-\frac{T_{k} T_{l} a^{i k} a^{j l}}{\|\mathbf{T}\|^{2}} \boldsymbol{\rho}_{i} \otimes \boldsymbol{\rho}_{j}\right]\left(\left(-\epsilon_{T} a_{m n}+\Gamma_{m n}^{r} T_{r}\right) \dot{\xi}^{n}-h_{m}^{r} T_{r} \dot{\xi}^{3}\right) \boldsymbol{\rho}^{m}=} \\
=\left(-\epsilon_{T} \dot{\xi}^{i}+a^{i k} T_{l} \Gamma_{k j}^{l} \dot{\xi}^{j}-h_{j}^{k} a^{i j} T_{k} \dot{\xi}^{3}\right) \boldsymbol{\rho}_{i}+ \\
+\frac{T_{k} T_{l} a^{i k}}{\|\mathbf{T}\|^{2}}\left(\epsilon_{T} \dot{\xi}^{l}-a^{j l} T_{m} \Gamma_{j n}^{m} \dot{\xi}^{n}+a^{j l} T_{m} h_{j}^{m} \dot{\xi}^{3}\right) \boldsymbol{\rho}_{i} .
\end{gathered}
$$

The time derivative of the normal force $N^{(n+1)}$ gives:

$$
\frac{d N^{(n+1)}}{d t}=\frac{d}{d t}\left(\epsilon_{N}\left|\xi^{3}\right|\right)=-\epsilon_{N} \dot{\xi}^{3}
$$

where the minus sign is a result from the conditions that the contact integral is computed only if $\xi^{3}<0$. Summarizing, we get:

$$
D_{v}\left(\delta W_{c}^{T}\right)=
$$

$$
\begin{aligned}
& \int_{s}\left(-\frac{\epsilon_{N} \mu \dot{\xi}^{3} T_{i} \delta \xi^{i}}{\|\mathbf{T}\|}-\right. \frac{\epsilon_{T} \mu|N| a_{i j} \dot{\xi}^{i} \delta \xi^{j}}{\|\mathbf{T}\|} \\
&+\frac{\mu|N| T_{k} \Gamma_{i j}^{k} \dot{\xi}^{j} \delta \xi^{i}}{\|\mathbf{T}\|}-\frac{\mu|N| T_{i} h_{j}^{i} \dot{\xi}^{3} \delta \xi^{j}}{\|\mathbf{T}\|} \\
&+\frac{\mu|N| T_{s} T_{l} \delta \xi^{s}}{\|\mathbf{T}\|^{3}}\left(\epsilon_{T} \dot{\xi}^{l}-a^{j l} T_{m} \Gamma_{j n}^{m} \dot{\xi}^{n}+a^{j l} T_{m} h_{j}^{m} \dot{\xi}^{3}\right)
\end{aligned}
$$




$$
\begin{gathered}
-\frac{\mu|N| T_{i}}{\|\mathbf{T}\|}\left[\left(\left(\delta \mathbf{r}_{s}-\delta \boldsymbol{\rho}\right) a^{i l} a^{j k} \boldsymbol{\rho}_{k} \otimes \boldsymbol{\rho}_{l} \mathbf{v}_{j}+\delta \boldsymbol{\rho}_{, j} a^{i k} a^{j l} \boldsymbol{\rho}_{k} \otimes \boldsymbol{\rho}_{l}\left(\mathbf{v}_{s}-\mathbf{v}\right)\right)\right. \\
+h^{i j}\left(\delta \mathbf{r}_{s}-\delta \boldsymbol{\rho}\right) \cdot\left(\boldsymbol{\rho}_{j} \otimes \mathbf{n}+\mathbf{n} \otimes \boldsymbol{\rho}_{j}\right)\left(\mathbf{v}_{s}-\mathbf{v}\right)+ \\
\left.\left.+h_{n}^{i} \dot{\xi}^{3} \delta \xi^{n}-\Gamma_{k j}^{i} \dot{\xi}^{j} \delta \xi^{k}\right]\right) d s .
\end{gathered}
$$

The sum of the parts $(106 a)$ and $(106 e)$ is zero. After some tensor algebra the other parts can be grouped into the following form:

$$
\begin{gathered}
D_{v}\left(\delta W_{c}^{T}\right)= \\
-\int_{s}\left(\left(\delta \mathbf{r}_{s}-\delta \boldsymbol{\rho}\right) \frac{\epsilon_{N} \mu T_{i} a^{i j}}{\|\mathbf{T}\|} \boldsymbol{\rho}_{j} \otimes \mathbf{n}\left(\mathbf{v}_{s}-\mathbf{v}\right)\right) d s \\
-\int_{s}\left(\left(\delta \mathbf{r}_{s}-\delta \boldsymbol{\rho}\right) \frac{\epsilon_{T} \mu|N| a^{i j}}{\|\mathbf{T}\|} \boldsymbol{\rho}_{i} \otimes \boldsymbol{\rho}_{j}\left(\mathbf{v}_{s}-\mathbf{v}\right)\right) d s \\
+\int_{s}\left(\left(\delta \mathbf{r}_{s}-\delta \boldsymbol{\rho}\right) \frac{\epsilon_{T} \mu|N| T_{i} T_{j} a^{i k} a^{j l}}{\|\mathbf{T}\|^{3}} \boldsymbol{\rho}_{k} \otimes \boldsymbol{\rho}_{l}\left(\mathbf{v}_{s}-\mathbf{v}\right)\right) d s \\
-\int_{s} \frac{\mu|N| T_{i}}{\|\mathbf{T}\|}\left(\left(\delta \mathbf{r}_{s}-\delta \boldsymbol{\rho}\right) a^{i l} a^{j k} \boldsymbol{\rho}_{k} \otimes \boldsymbol{\rho}_{l} \mathbf{v}_{j}+\delta \boldsymbol{\rho}_{, j} a^{i k} a^{j l} \boldsymbol{\rho}_{k} \otimes \boldsymbol{\rho}_{l}\left(\mathbf{v}_{s}-\mathbf{v}\right)\right) d s \\
+\int_{s}\left(\frac{\mu|N| T_{i}}{\|\mathbf{T}\|} h^{i j}\left(\delta \mathbf{r}_{s}-\delta \boldsymbol{\rho}\right) \cdot\left(\boldsymbol{\rho}_{j} \otimes \mathbf{n}+\mathbf{n} \otimes \boldsymbol{\rho}_{j}\right)\left(\mathbf{v}_{s}-\mathbf{v}\right)\right) d s \\
+\int_{s} \frac{\mu|N| T_{s} T_{l} \delta \xi^{s}}{\|\mathbf{T}\|^{3}}\left(-a^{j l} T_{m} \Gamma_{j n}^{m} \dot{\xi}^{n}+a^{j l} T_{m} h_{j}^{m} \dot{\xi}^{3}\right) d s .
\end{gathered}
$$

The matrix consists then of a constitutive non-symmetric part (107), a constitutive symmetric part $(107 a)$ and $(107 b)$, a symmetric rotational part $(107 c)$, a symmetric curvature part (107 $d)$ and a non-symmetric part curvature part $(107 e)$ which is preserved for curved surfaces. All geometrical parameters are computed for the master surface.

Remark 2. One can find from comparison with Peric and Owen [20], that they have considered the tangent matrix which is represented by the the main parts of the full tangent matrix. 


\section{Global solution scheme. Summary of the results.}

Summarizing the theoretical discussion about the covariant description, we present the global solution scheme for the numerical implementation in Table 1 and 2. All parts of tangent matrices contain either a term $\left(\delta \mathbf{r}_{s}-\delta \boldsymbol{\rho}\right)$, or a term $\delta \boldsymbol{\rho}_{, j}$, resp. terms $\left(\mathbf{v}_{s}-\mathbf{v}\right)$ and $\mathbf{v}_{, j}$, and, therefore, can be algorithmically computed. For discretization of any surface only two position matrices $\mathbf{A}$ and $\mathbf{A}_{\xi}$ are necessary. The proposed approach has been implemented in FEAP code see [27], "solid-shell" elements are used for modelling of elastic structures, see [7] and [8]. For the details of the finite element implementation we refer to Konyukhov and Schweizerhof [10]. 


\section{Table 1: Global solution scheme. Summary of the results for numerical implementation.}

1. Initialization of convective coordinates $\xi^{i}$.

The projection procedure in eqns. $(23,24,25)$ with no external loads gives $\xi_{(0)}^{i}$.

2. Loop over load increments and Newton iterations

for the contact integral

$\delta W_{c}=\int_{s} N \delta g d s+\int_{s} T_{j} \delta \xi^{j} d s \quad$ where $\delta \xi^{j}=a^{i j}\left(\delta \mathbf{r}_{s}-\delta \boldsymbol{\rho}\right) \cdot \boldsymbol{\rho}_{i}$

3. Loop over all contact elements and all contact points

- compute projection points $\xi_{(n)}^{i}$ eqns. $(23,24,25)$

- Check penetration $g=\left(\mathbf{r}_{s}-\boldsymbol{\rho}\right) \cdot \mathbf{n}$. If $g>0$ then exit loop 3 .

- Compute contact tractions and corresponding tangent matrices at each contact point.

Normal traction: $N=\epsilon_{N} g$

Tangent matrix $\mathbf{K}^{\mathbf{N}}$ for normal traction is defined via

$$
\begin{aligned}
& \int_{s} \epsilon_{N}\left(\delta \mathbf{r}_{s}-\delta \boldsymbol{\rho}\right) \cdot(\mathbf{n} \otimes \mathbf{n})\left(\mathbf{v}_{s}-\mathbf{v}\right) d s-\int_{s} \epsilon_{N} g\left(\delta \boldsymbol{\rho}_{, j} \cdot a^{i j}\left(\mathbf{n} \otimes \boldsymbol{\rho}_{i}\right)\left(\mathbf{v}_{s}-\mathbf{v}\right)+\right. \\
& \left.\quad+\left(\delta \mathbf{r}_{s}-\delta \boldsymbol{\rho}\right) \cdot a^{i j}\left(\boldsymbol{\rho}_{j} \otimes \mathbf{n}\right) \mathbf{v}_{, i}\right) d s-\int_{s} \epsilon_{N} g\left(\delta \mathbf{r}_{s}-\delta \boldsymbol{\rho}\right) \cdot h^{i j}\left(\boldsymbol{\rho}_{i} \otimes \boldsymbol{\rho}_{j}\right)\left(\mathbf{v}_{s}-\mathbf{v}\right) d s
\end{aligned}
$$

Tangent traction $T_{i}$ is defined via the return-mapping algorithm.

Trial step

$$
T_{i}^{(n+1)}=T_{k}^{(n)} a_{(n)}^{k j}\left(\boldsymbol{\rho}_{j}{ }^{(n)} \cdot \boldsymbol{\rho}_{i}{ }^{(n+1)}\right)-\epsilon_{T} \cdot\left\{\begin{array}{c}
\left(\Delta \boldsymbol{\rho} \cdot \boldsymbol{\rho}_{i}\right) \text { for node-to-surface (NTS) and } \\
\text { surface-to-surface (STS) approaches, where } \\
\Delta \boldsymbol{\rho}=\left.\boldsymbol{\rho}_{C^{(n+1)}}\right|_{\xi_{(n+1)}^{1}}, \xi_{(n+1)}^{2}-\left.\left(\boldsymbol{\rho}_{C^{(n)}}+\mathbf{u}_{\left.C^{(n)}\right)}\right)\right|_{\left.\xi_{(n)}^{1}\right)}, \xi_{(n)}^{2} \\
-\left(\mathbf{u} \cdot \boldsymbol{\rho}_{i}\right) \text { for segment-to-analytical surface (STAS) approach }
\end{array}\right.
$$

Coulomb friction law:

$\Phi_{(n+1)}=\sqrt{T_{i}^{(n+1)} T_{j}^{(n+1)} a_{(n+1)}^{i j}}-\mu N^{(n+1)}$

Return-mapping step see Table 2.

- Compute residual $\mathbf{R}$ from the contact integral in 2

- Compute the full contact tangent matrix $\mathbf{K}=\mathbf{K}^{\mathbf{N}}+\mathbf{K}^{\mathbf{T}}$ 
Table 2: Return-mapping scheme and tangent matrices for tangential traction.

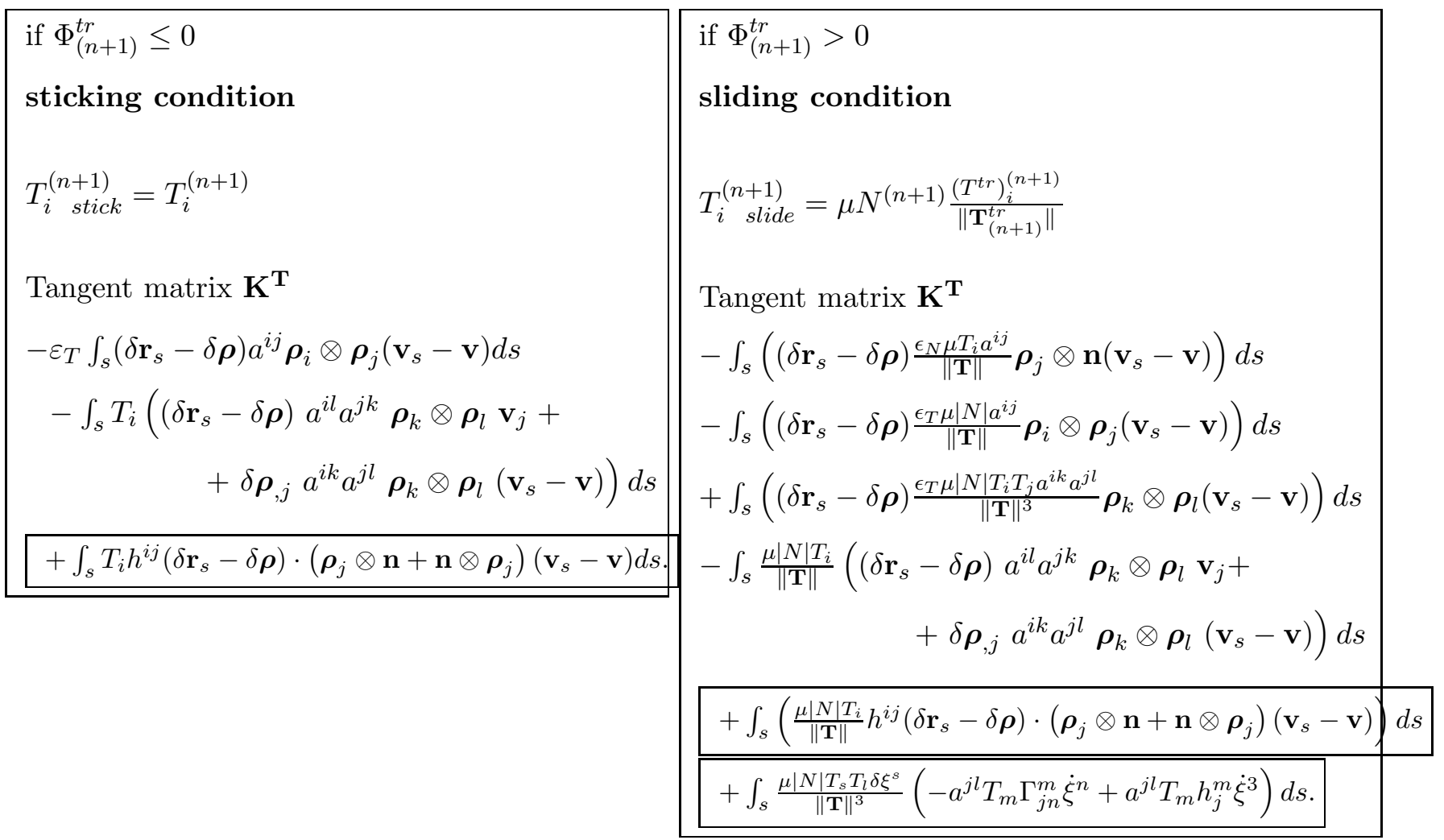

Remark. Curvature parts in boxes (Table 1 and 2) can be omitted with very little loss of efficiency 


\section{$6 \quad$ Numerical examples}

\subsection{Sliding of a block with a linear approximation of the contact surfaces.}

During the solution of the frictional problem, it is necessary to solve the evolution equation (74) with a return-mapping algorithm, as described in (77), (78) and (79). As was mentioned in Remark 2, it is important to know the value of coordinate increments $\Delta \xi^{i}$ and, therefore, displacement increments $\Delta u^{i}$ in order to capture the "sticking-sliding" zone correctly. As we can see later from the numerical examples this fact leads to a separation of the contact problem into two different types. As a representative example for a-priori estimation of the value of incremental displacements, the stresses in an infinite layer have to be considered, see Fig. (3). Both a vertical displacement $h$ and a horizontal displacement $u$ are applied at the upper boundary. During the deformation the rectangle $A B C D$ is changing into a parallelogram $A B_{1} C_{1} D$. Under the assumption of linear elasticity and a plane strain deformation, the stresses in the layer are obtained via superposition of the normal compressive stress $\sigma$ and the pure shear stress $\tau$ :

$$
\sigma=\varepsilon \frac{E}{1-\nu^{2}}=\frac{h}{b} \frac{E}{1-\nu^{2}} ; \quad \tau=\gamma G=\frac{u}{b} \frac{E}{2(1+\nu)} .
$$

Now we assume Coloumb friction with $\mu$ as a friction coefficient at the lower boundary. Sliding starts if the condition $\tau=\mu \sigma$ is fulfilled. Thus, the condition of sticking of the thin layer can estimated by the following ratio:

$$
\frac{\gamma(1-\nu)}{2 \varepsilon} \leq \mu
$$

from which we obtain the threshold value of the horizontal displacement $u$ :

$$
u_{c r}=\frac{2 \mu h}{1-\nu}
$$

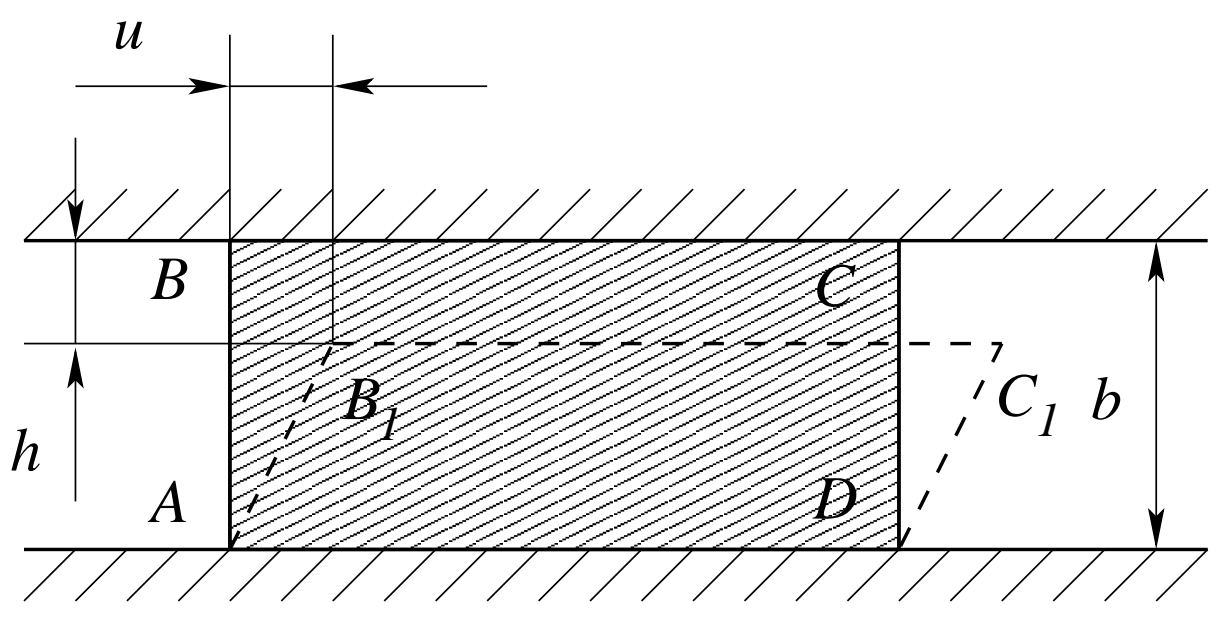

Figure 3: Plane deformation of a layer.

One can see from the infinite layer, that sliding starts immediately at the complete lower boundary. However, though this is not a case for a finite dimensional block, or an arbitrarily thin layer, where a developing zone of sticking and sliding exists, eqns. (109) and (110) can be used as a rough estimation of the presence of the sticking condition, and, therefore, for the estimation of the displacement increments. 


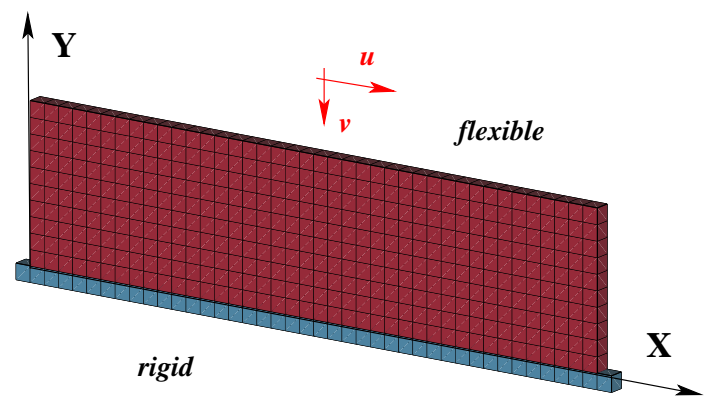

Figure 4: Sliding block on the base. Meshed surfaces. STS contact approach.

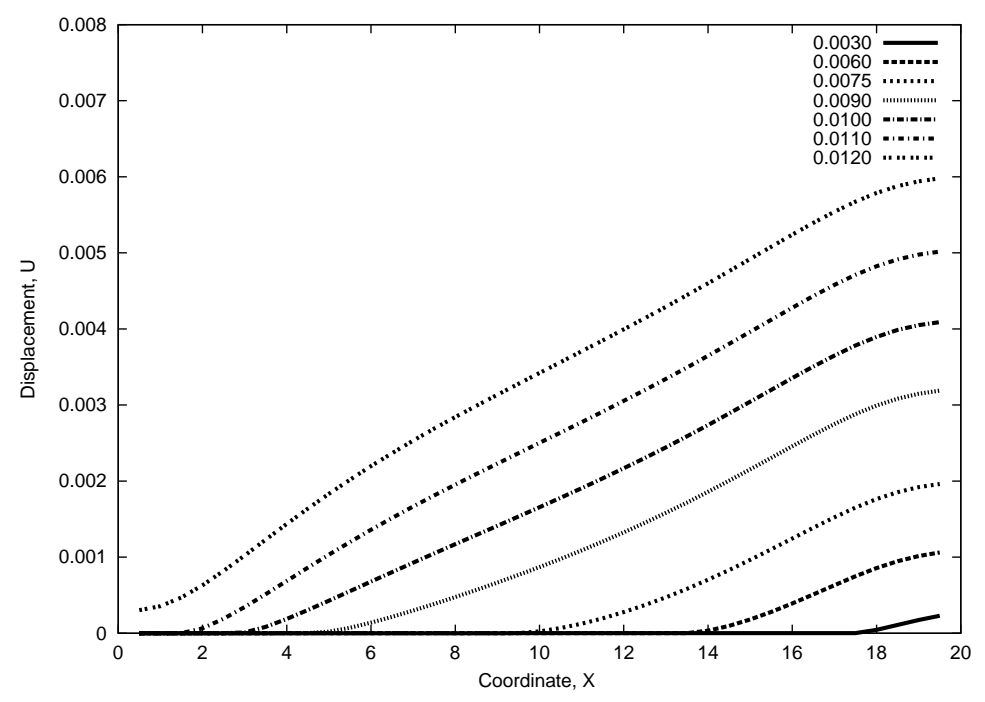

Figure 5: Horizontal displacements of the contact surface for various states of the displacement loading.

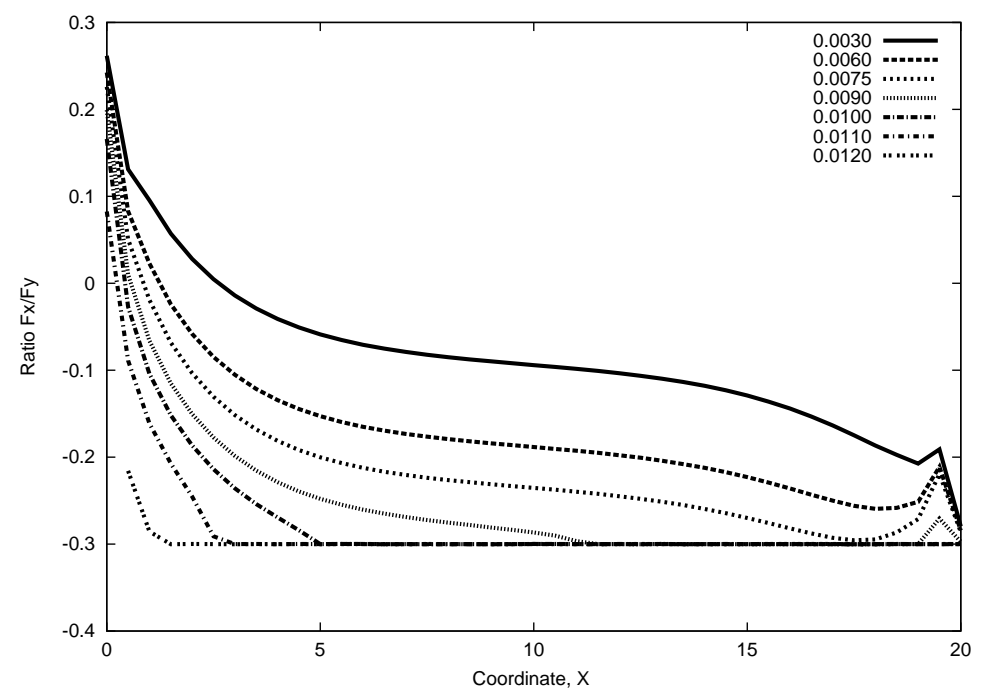

Figure 6: Reaction forces ratio $F_{x} / F_{y}$ on the contact surface for various states of the displacement loading. 
As an example for the computation, we consider a rectangular block (Fig. 4) with the following parameters: elasticity modulus $E=2.1 \cdot 10^{4}$, Poisson ratio $\nu=0.3$, length $a=20$, height $b=5$, thickness $c=0.5$. The dimension system is assumed to be consistent. The lower supplementary block is added to model a rigid base. The Coloumb friction with a coefficient $\mu=0.3$ is specified between two bodies. The contact surface of the upper block is assumed to be a "master", while the upper surface of the lower block is a "slave" surface within the "segment-to-segment" approach. The penalty parameters are chosen as $\varepsilon_{N}=\varepsilon_{T}=2.1 \cdot 10^{6}$.

Since the problem is path-dependent, we will investigate a case when displacements at the upper edge are applied in two steps: at the first step, a vertical displacement $v=-7.0 \cdot 10^{-3}$ is applied, then, a horizontal displacement is applied incrementally. Here, we should mention that initial conditions for the history variables are defined at the zero load step with zero external loads, i.e. the initial projection points are the sticking points, see step 1 in Table 1. An estimation of the critical horizontal displacement in eqn. (110) gives $u_{c r}=6.0 \cdot 10^{-3}$, so in order to capture the sticking-sliding zone we choose a displacement increment $\Delta u=2.5 \cdot 10^{-4}$ and apply it in 100 load steps. Our aim in the first computation is to show the development of the sticking-sliding zone. In order to verify this zone carefully we will consider a plot of the horizontal displacements and a plot of the reaction forces ratio on the boundary $F_{x} / F_{y}$. Of course, this zone is precisely specified by the return-mapping algorithm, but we are interested in various parameters. Fig. 5 contains the spatial distribution of the horizontal displacements at the lower boundary, if the following displacements $u=3.0 \cdot 10^{-3} ; 6.0 \cdot 10^{-3} ; 7.5 \cdot 10^{-3} ; 9.0 \cdot 10^{-3} ; 10.0 \cdot 10^{-3} ; 11.0 \cdot 10^{-3} ; 12.0 \cdot 10^{-3}$ are applied at the upper boundary. As shown in the corresponding reaction forces ratio diagram in Fig. 6, sliding starts from $u=7.5 \cdot 10^{-3}$, when the ratio $F_{x} / F_{y}=-0.3$ is reached. The block is considered to be sliding at the full lower boundary, when the applied displacement reaches the value $u=11.0 \cdot 10^{-3}$. We can also conclude that the estimation of the threshold displacement given by eqn. (110) is a good approximation.

The spreading of the zone of sliding is found to be within a relatively short interval of loading. In some practical problems, as e.g. metal forming, the energy loss due to large sliding can be more important. Assume for the next discussion that stresses are approximated by eqn. (108) for the finite-dimensional block with size $A D=a$, e.g. for a very long block. Then the elastic energy accumulated at the critical state in the block has the following form:

$$
E_{e l}=\frac{\sigma \varepsilon a b}{2}+\frac{\tau \gamma a b}{2}=\frac{E a h^{2}}{2 b\left(1-\nu^{2}\right)}\left[1+\frac{2 \mu^{2}}{1-\nu}\right] .
$$

If the sliding process is developing, when the block is dragged along the distance $l$, then the work of the critical sliding stresses $\tau_{s l}$, is evaluated as:

$$
E_{s l}=\frac{\tau_{s l} a l}{2}=\frac{E a h l \mu}{b\left(1-\nu^{2}\right)} .
$$

It is obvious, that during the large sliding a thin layer along a relatively large distance $l$, dissipation of energy due to sliding, eqn. (112) can be rather important then initial threshold value. Thus, frictional problems can be subdivided into two problems:

a) compute the global threshold value for sliding and the development of the distribution of the sticking-sliding zone;

b) compute forces which are necessary to drag the structure under the assumption of full sliding.

Obviously, for the first problem the evolution equation (74) must be computed with small steps within the return-mapping algorithm, but for the second problem the sticking zone is out of interest and for the analysis relatively large steps can be taken. Such problems are certainly 
present in forming processes with large plastic deformations. In order to show an example for the problem type b), another analysis is performed with a displacement increment $\Delta u=12.0 \cdot 10^{-3}$, which is even larger then the critical one and corresponds to the developed sliding zone, see Fig. 5. In order to compare the influence of the various parts of the tangent matrix we compute two cases 1) with the full tangent matrix;

2) only with the main part of the tangent matrix.

The penalty parameter is chosen as $\varepsilon_{N}=\varepsilon_{T}=2.1 \cdot 10^{5}$. Table 3 shows the comparison of the numerical results between both cases by the number of iterations per load step. As we can see in the developed sliding region the full matrix in comparison with only the main matrix leads to a reduction of the number of equilibrium iterations per load step from 4 to 3 . We should mention that for the previous example during the incremental horizontal loading there is no difference between the number of equilibrium iterations for both cases. Obviously this is due to the fairly small load steps. Thus, as expected, keeping all parts of the matrix appears to be only necessary in the case of large load increments.

Fig. 7 shows the spatial distribution of the relative horizontal displacements $u-u_{\text {applied }}$ at the lower boundary if the displacement at the upper boundary is taken exemplarily as $u=0.012$, then $u=0.048$ and finally $u=0.120$. It is obvious, that the relative horizontal displacements hardly change during the fairly large sliding process.

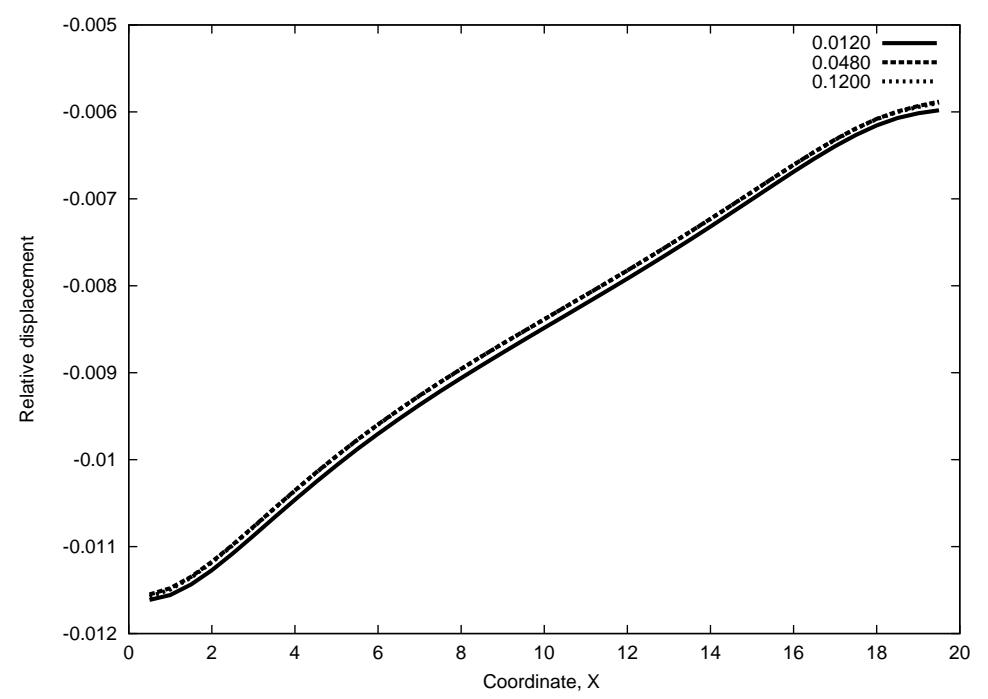

Figure 7: Relative horizontal displacements of the contact surface for various states of the displacement loading. 


\begin{tabular}{|c|c|c|c|c|c|}
\hline \multicolumn{3}{|c|}{ Case 1 } & \multicolumn{3}{c|}{ Case 2 } \\
\hline No. l.s. & $\begin{array}{c}\text { No. } \\
\text { it./l.s. }\end{array}$ & $\begin{array}{c}\text { Cum. } \\
\text { No. it. }\end{array}$ & No. l.s. & $\begin{array}{c}\text { No. } \\
\text { it./l.s. }\end{array}$ & $\begin{array}{c}\text { Cum. } \\
\text { No. it. }\end{array}$ \\
\hline 1 & 4 & 4 & 1 & 5 & 5 \\
\hline 2 & 6 & 10 & 2 & 6 & 11 \\
\hline 3 & 5 & 15 & 3 & 5 & 16 \\
\hline $4-20$ & 3 & 66 & $4-20$ & 4 & 84 \\
\hline
\end{tabular}

Table 3: Sliding of a block. Bilinear elements. Segment-to-segment contact approach. Influence of various contact stiffness parts on convergence. Case 1: full matrix; case 2: only main matrix. Comparison of no. of iterations in all load steps (l.s.)

\subsection{Sliding of a block. Quadratical approximation of the contact surfaces.}

Since general smoothing techniques for contact surfaces are out of the scope of this article, in this example we will use contact elements with quadratical approximation of the master surface together with a specially chosen geometry of both contact bodies in order to preserve $C^{1}$-continuity of the contact surfaces. Namely, we consider contact between a parabolical block sliding on a parabolical cylindrical base, see Fig. 8. The block is meshed with 18-node solid-shell elements with density $20 \times 1 \times 5$. Both master and slave contact surface geometries are satisfying the equation

$$
z=c \cdot x^{2}, \text { with } \quad c=0.03 .
$$

The contact is modeled by the node-to-surface approach with the master surface from the parabolical block. The parabolical slave surface of the fixed base is represented by slave nodes with the same mesh density as the master, which are not shown in Fig. 8. The geometrical parameters are $H=5, L=10$; the material is linear elastic with Young's modulus $E=2.1 \cdot 10^{4}$, Poisson ratio $\nu=0.3$, Coulomb friction coefficient $\mu=0.3$.

In the case of contact with a curvilinear surface, even with homogeneous loading, zones with sticking and sliding can be present. One can expect from the rigid body mechanics that the sliding zone during vertical loading $w$ in the current example is satisfying the following condition $|x|>5$. From the friction cone for the parabolical cylinder follows that: $\left.\tan \alpha\right|_{x=5}=z^{\prime}=\left.2 \cdot 0.03 x\right|_{x=5}=0.3$. In order to inspect this effect in the deformable body, we apply at the upper edge the vertical displacement $w=0.007$ in 7 load steps. In Fig.9 the distribution of the tangent displacement $u_{\tau}=u_{x} \cos \alpha+u_{y} \sin \alpha$ over $x$ on the contact surface is depicted, where now and for the next example the angle $\alpha$ is computed in the reference configuration. For the further discussion we will distinguish based on the OZ axis, the left sliding zone with negative displacements and the right sliding zone with positive displacements. The tangential displacements from both zones are directed towards the $\mathrm{OZ}$ axis, therefore the distribution looks mirror-symmetric. One can see that the sticking zone is approximately satisfying the condition $|x|<3$.

Equidistant motion on a cylinder. As continuation of the numerical example, we choose an equidistant motion of the upper edge of the parabolical block at the distance $h$ from the generatrix of the parabolical base. The curve $\mathbf{r}$ of this motion satisfies the following equation:

$$
\mathbf{r}=\boldsymbol{\rho}+h \mathbf{n},
$$




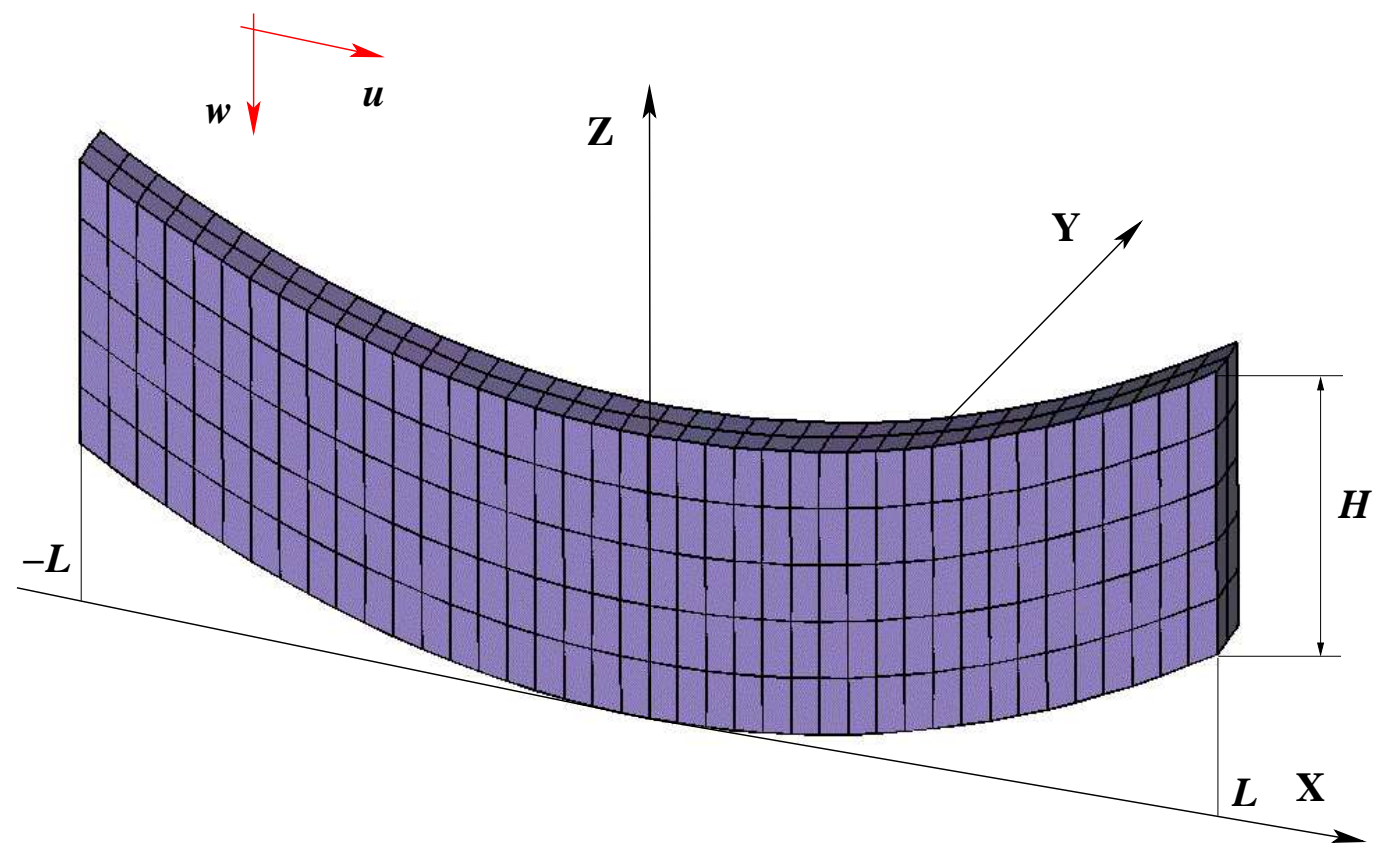

Figure 8: Sliding of a parabolical block on a parabolical base. Meshed block. NTS contact approach.

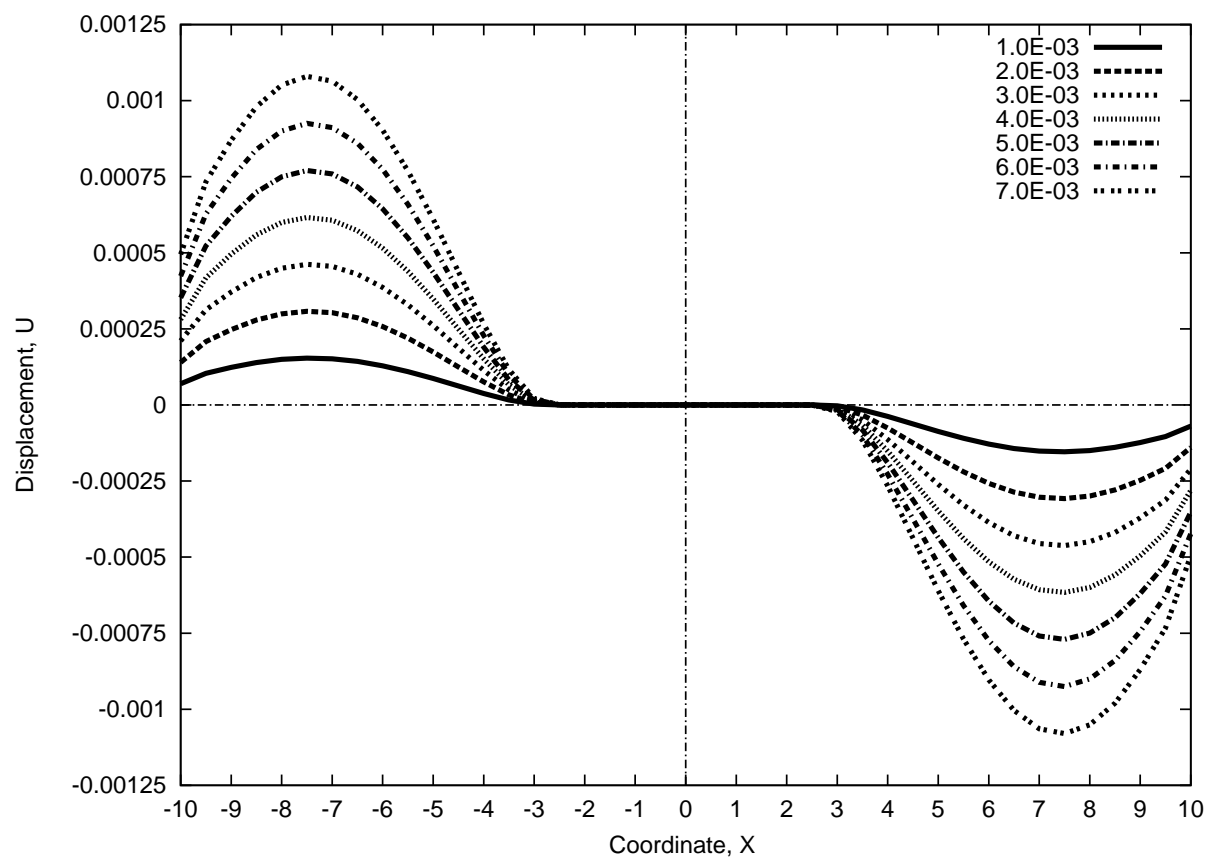

Figure 9: Distribution of the tangential displacements in OX direction. Parabolical cylinder. NTS contact approach. Incremental vertical loading. 
which for the parabola (113) can be written as:

$$
\mathbf{r}=\left\{\begin{array}{c}
x \\
0 \\
c x^{2}
\end{array}\right\}+\frac{h}{\sqrt{1+4 c^{2} x^{2}}}\left\{\begin{array}{c}
-2 c x \\
0 \\
1
\end{array}\right\}
$$

where $\mathbf{n}$ is a normal on the initial curve, and $h$ is an initial vertical displacement. From eqn. (115) it is clear that the trajectory of the body is no longer a parabola. If the curvature of the cylinder is small, i.e. $c<<1$, then we consider a Taylor expansion with a linear term for the first coordinate and with a quadratic term for the second coordinate. Thus, we obtain as a first approximation of the trajectory in eqn. (115) a parabolical motion in the form:

$$
\mathbf{r}=\left\{\begin{array}{c}
x(1-2 c h) \\
0 \\
h+c x^{2}(1-2 c h)
\end{array}\right\} .
$$

In this displacement driven problem the parabolical block is moving in the $\mathrm{X}-\mathrm{Z}$ plane, providing an approximately constant compression.

Next, the loading is applied in two steps also: the first step is a vertical loading with $w=$ $-h=0.007$, then both a horizontal and a vertical loading are incrementally applied at the upper edge with $\Delta u=\Delta x=2.5 \cdot 10^{-5}$ according to eqn. (116), providing the equidistant motion of the parabolical block. Now, two phases of the development of the sticking-sliding zone can be observed. The first phase corresponds to the situation when the right sliding zone disappears during horizontal loading, as presented in Fig. 10 for the following load steps: $u=1.0 \cdot 10^{-3}$, $2.0 \cdot 10^{-3}, 3.0 \cdot 10^{-3}, 5.0 \cdot 10^{-3}$. Fig. 11 a) shows scaled deformed and undeformed states when only vertical displacements are applied and, therefore, the two sliding zones are symmetric. The configuration with the vanished sliding zone on the right side is presented in Fig. $11 \mathrm{~b}$ ). This moment can be detected also from the reaction forces ratio diagram $F_{\tau} / F_{n}$ in Fig. 13, where the right part of the sliding zone is also disappearing with $F_{\tau} / F_{n}=0.3$. The second phase is the spreading of the left sliding zone through the contact surface shown in Fig. 12 exemplarily for $u=5.0 \cdot 10^{-3}, 10.0 \cdot 10^{-3}, 15.0 \cdot 10^{-3}, 20.0 \cdot 10^{-3}, 25.0 \cdot 10^{-3}, 30.0 \cdot 10^{-3}$. Here the zone without contact is detected as a zone with zero normal nodal forces $f_{n}=0$ starting at a loading with $u \geq 1.52 \cdot 10^{-2}$. These sub-zones are marked with thicker lines in Fig. 12. 


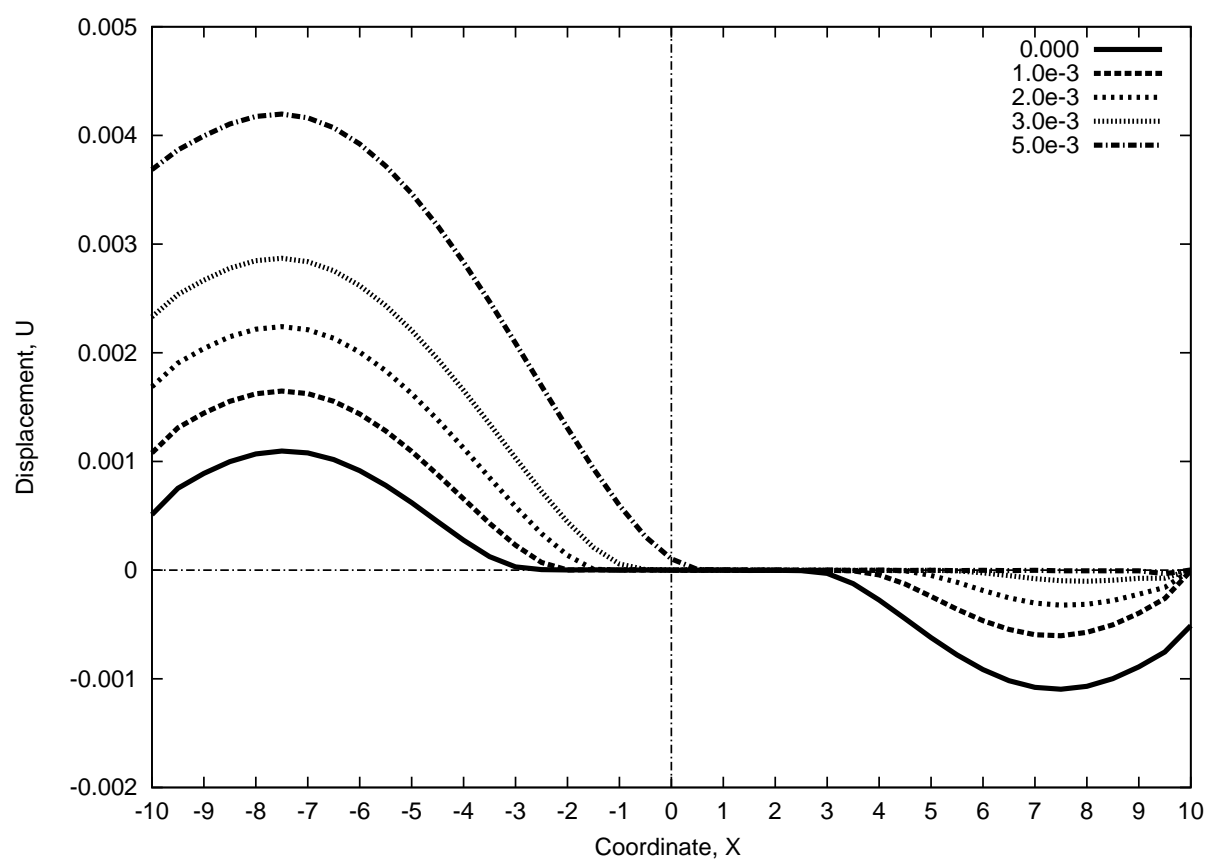

Figure 10: Parabolical cylinder. Horizontal loading. Distribution of the tangential displacements in X direction. Phase 1 — vanishing right sliding zone.

Again we now compare the influence of the various parts of the tangent matrix on the convergence rate when the applied displacements correspond to the developed sliding. Namely, the load is applied in 20 load steps with the displacement increment $\Delta u=4.0 \cdot 10^{-2}$, providing a fully developed sliding motion from the first step on. The following cases are shown in table 4:

1) full tangent matrix;

2) without curvature parts;

3) only with main part of the tangent matrix.

We see that excluding the curvature matrix leads to a minor reduction of the convergence, while excluding the rotational part too causes a considerable increase of the number of equilibrium iterations per load step. We should also mention that during the analysis of the threshold value before full sliding the number of equilibrium iterations remains the same for each case due to a small load step. Thus, the computation with the rotational part is more important for the developed sliding problem of type $\mathbf{b}$. 


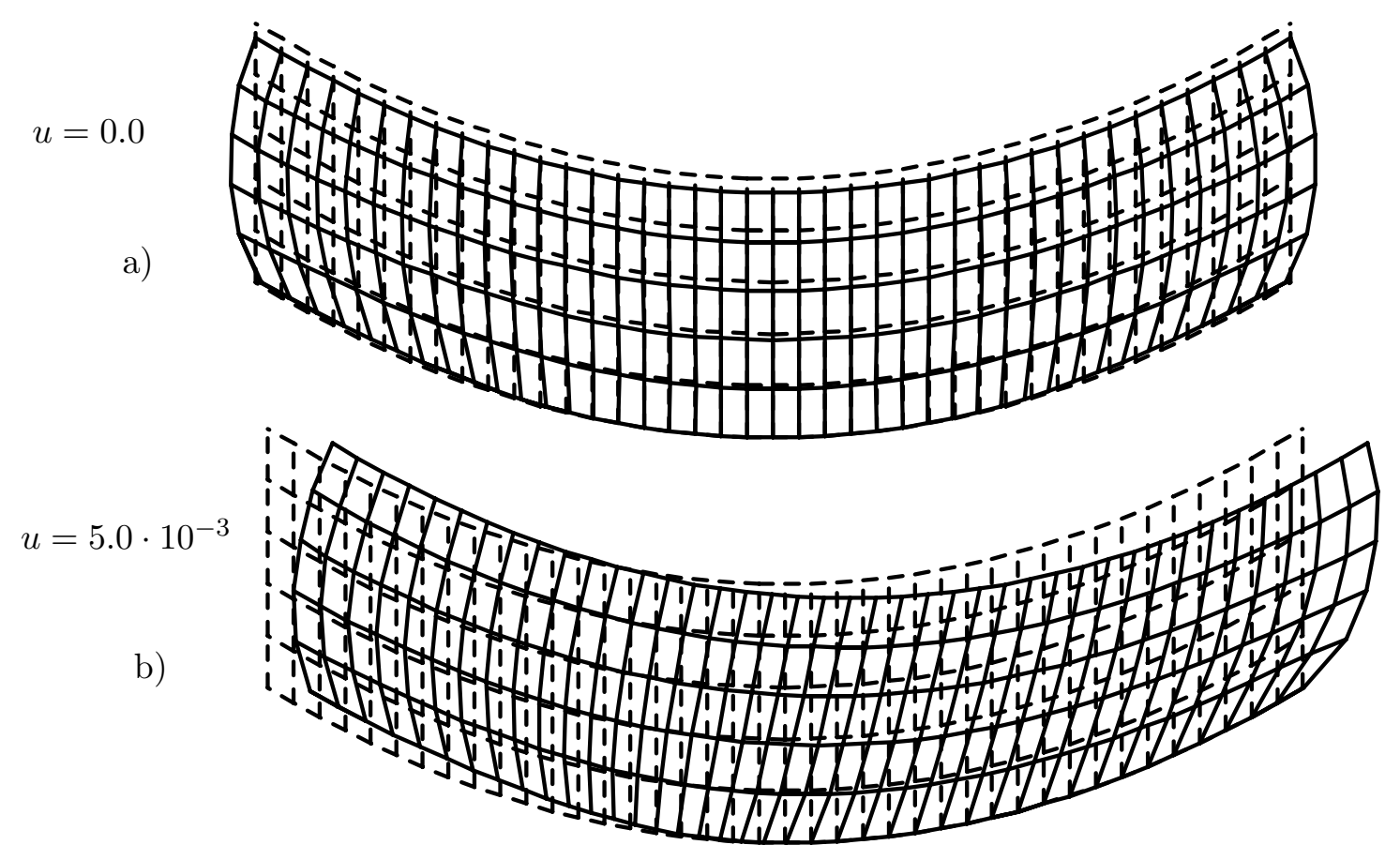

Figure 11: Parabolical cylinder. Initial vertical loading by displacement $w_{0}=0.007$ of the top edge. Undeformed and deformed states with in addition applied horizontal displacement a) $u=0.0$ - two sliding zones; b) $u=5.0 \cdot 10^{-3}$ - sliding zone only on the left. (Displacements scaled: 250 times in $\mathrm{x}$-direction, 40 times in $\mathrm{z}$-direction.)

\begin{tabular}{|c|c|c|c|c|c|c|c|c|}
\hline \multicolumn{3}{|c|}{ Case 1 } & \multicolumn{3}{c|}{ Case 2 } & \multicolumn{3}{c|}{ Case 3 } \\
\hline No. l.s. & $\begin{array}{c}\text { No. } \\
\text { it./l.s. }\end{array}$ & $\begin{array}{c}\text { Cum. } \\
\text { No. it. }\end{array}$ & No. l.s. & $\begin{array}{c}\text { No. } \\
\text { it./l.s. }\end{array}$ & $\begin{array}{c}\text { Cum. } \\
\text { No. it. }\end{array}$ & No. l.s. & $\begin{array}{c}\text { No. } \\
\text { it./l.s. }\end{array}$ & $\begin{array}{c}\text { Cum. } \\
\text { No. it. }\end{array}$ \\
\hline 1 & 6 & 6 & 1 & 6 & 6 & $1-9$ & 6 & 54 \\
\hline 2 & 5 & 11 & 2 & 5 & 11 & $10-12$ & 7 & 75 \\
\hline 3 & 5 & 16 & 3 & 5 & 16 & $13-15$ & 8 & 99 \\
\hline $4-18$ & 4 & 76 & $4-16$ & 4 & 68 & $15-17$ & 9 & 126 \\
\hline $19-20$ & 5 & 86 & $17-20$ & 3 & 88 & $18-20$ & 10 & 156 \\
\hline
\end{tabular}

Table 4: Full sliding of a parabolical block. Biquadratic elements. Node-to-segment contact approach. Influence of various contact stiffness parts on convergence. Case 1: full matrix; case 2: without curvature parts; case 3: only main matrix. Comparison of no. of iterations in all load steps (l.s.)

\subsection{Large sliding on a rigid parabolical cylinder}

As an example of a problem with a 3D spatial large sliding, we consider here a motion of a semi-circular cylinder on the surface of a parabolical cylinder in analogy to [11], see Fig. 14. The necessary details for the description of contact with rigid surfaces described by analytical functions is given in a short from [6].

\subsubsection{Contact with a surface described by analytical functions}

If a body contacts a rigid surface, the latter one is chosen as a "slave" surface in our description, but the integration is performed over the "master" surface. The rigid surface is then parameterized 


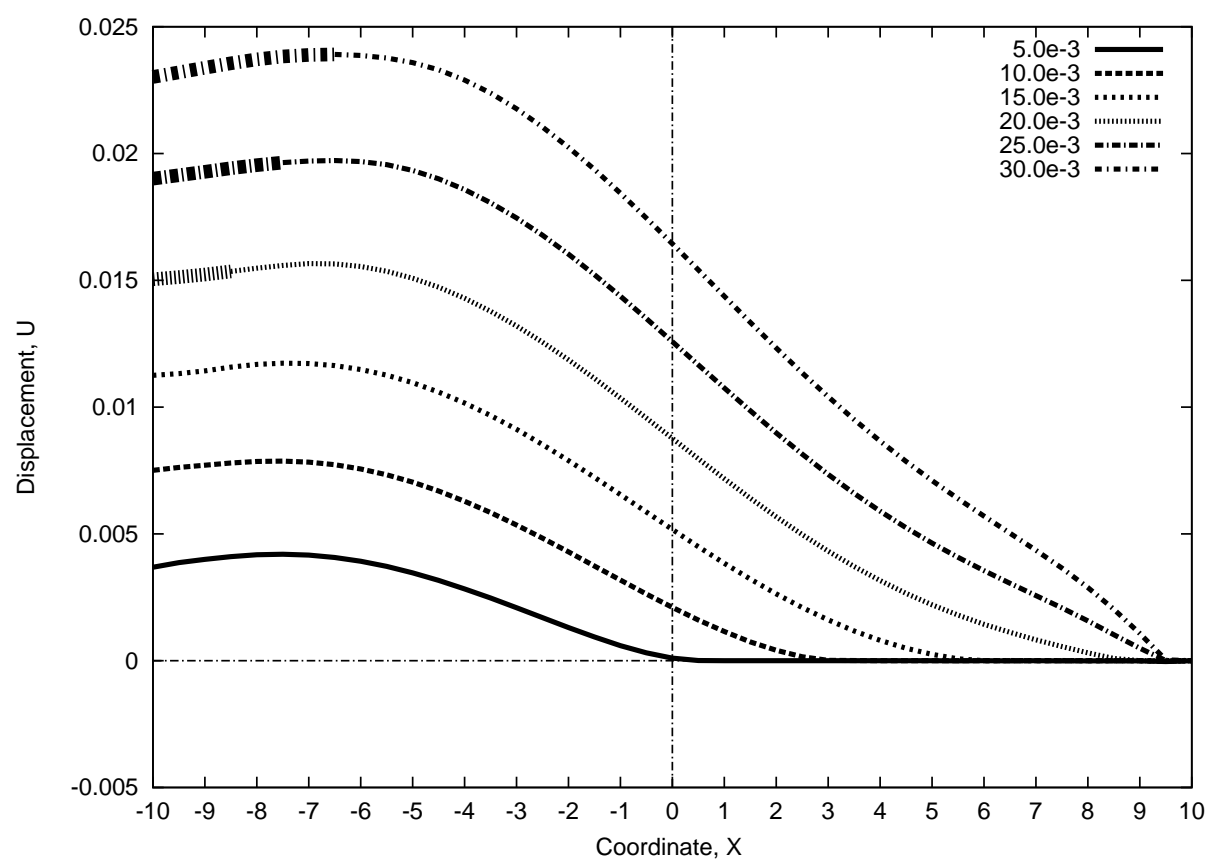

Figure 12: Parabolical cylinder. Horizontal loading. Distribution of the tangent displacement over $\mathrm{X}$-coordinate. Phase 2 - spreading of the left sliding zone.

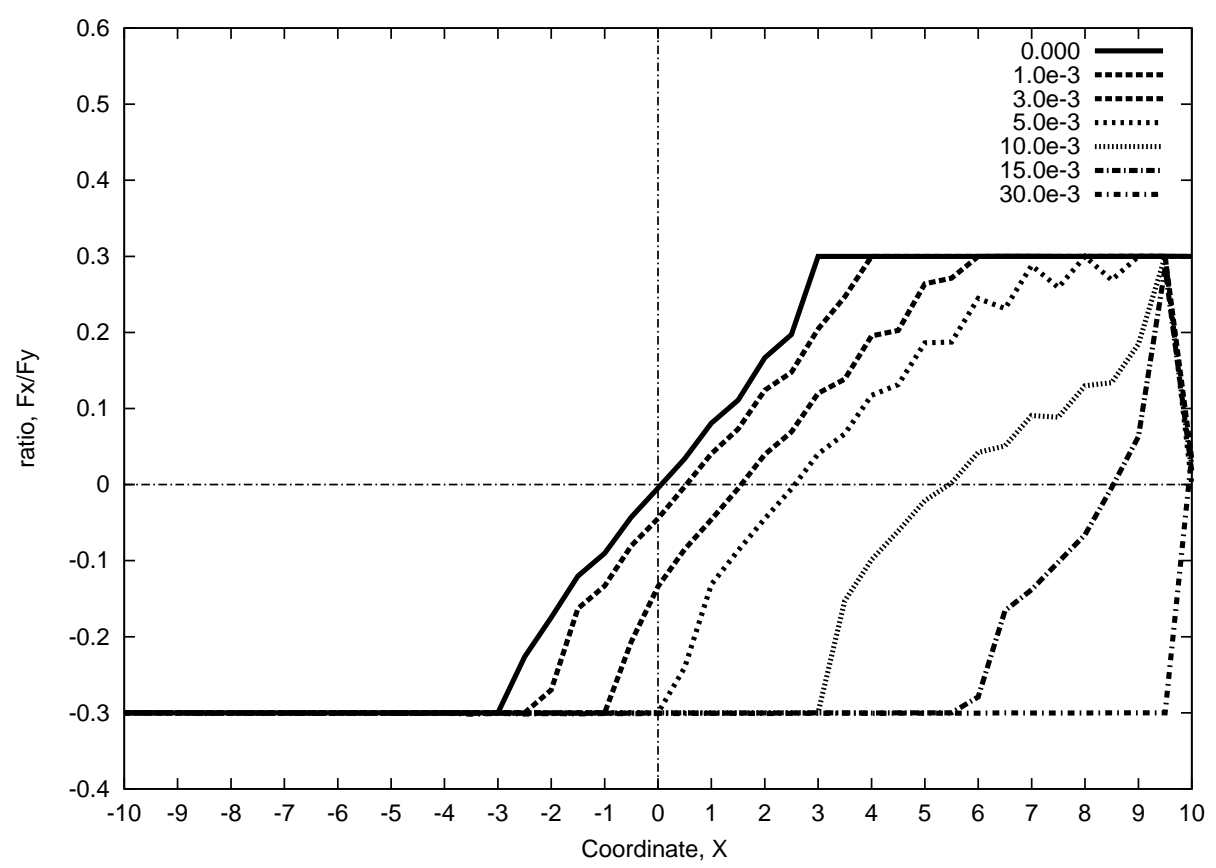

Figure 13: Parabolical cylinder. Horizontal loading. Reaction forces ratio $F_{\tau} / F_{n}$ on the contact surface for various states of loading.

by internal coordinates $\alpha^{1}, \alpha^{2}$. Then a point $\mathbf{r}$ of this surface has to satisfy eqn. (26) as a point in the local coordinate system of the contact element too. This condition leads to the following equation

$$
\mathbf{r}\left(\alpha^{1}, \alpha^{2}\right)=\boldsymbol{\rho}\left(\xi^{1}, \xi^{2}\right)+\xi^{3} \mathbf{n} .
$$


The 'slave' point projection procedure, which was necessary for the previous description with surface segments, now turns into the determination of the surface point defined by equation (117). Using a "segment-to-segment" type strategy for the computation of the contact integral, first integration points $\xi_{I}^{1}, \xi_{J}^{2}$ are defined on the "master" segment and then the corresponding internal coordinates $\alpha^{1}, \alpha^{2}$ of the rigid surface as well as the penetration $\xi^{3}$ are computed e. g. by the Newton method. For this algorithm we define a function $F\left(\alpha^{1}, \alpha^{2}, \xi^{3}\right)$ with the components given in eqn. (117)

$$
\mathbf{F}=\left[\begin{array}{c}
x_{s 1}-x_{1}-n_{1} \xi^{3} \\
x_{s 2}-x_{2}-n_{2} \xi^{3} \\
x_{s 3}-x_{3}-n_{3} \xi^{3}
\end{array}\right] \quad \text { with } \quad x_{i}=x_{i}\left(\xi^{1}, \xi^{2}\right)
$$

Its derivative with respect to the coordinates $\left(\alpha^{1}, \alpha^{2}, \xi^{3}\right)$ is:

$$
\mathbf{F}^{\prime}=\left[\begin{array}{ccc}
x_{s 1,1} & x_{s 1,2} & -n_{1} \\
x_{s 2,1} & x_{s 2,2} & -n_{2} \\
x_{s 3,1} & x_{s 3,2} & -n_{3}
\end{array}\right]
$$

Then, the Newton iteration procedure reads as follows for iteration step $n$ :

$$
\begin{aligned}
\Delta \boldsymbol{\alpha}_{n} & =\left[\begin{array}{c}
\Delta \alpha_{n}^{1} \\
\Delta \alpha_{n}^{2} \\
\Delta \xi_{n}^{3}
\end{array}\right]=-\left(\mathbf{F}^{\prime}\right)_{n}^{-1} \mathbf{F}_{n}, \\
\boldsymbol{\alpha}_{n+1} & =\boldsymbol{\alpha}_{n}+\Delta \boldsymbol{\alpha}_{n} .
\end{aligned}
$$

Parabolical cylinder. Consider a parabolical cylinder in the canonical form:

$$
\begin{gathered}
x_{s}=\alpha \\
y_{s}=c \alpha^{2} .
\end{gathered}
$$

The Newton procedure in eqn. (120) in this case is reduced to the definition of $\alpha$ from the following iterative expression:

$$
\alpha^{(n+1)}=\frac{c\left(\alpha^{(n)}\right)^{2} n_{1}+n_{1} x_{2}-n_{2} x_{1}}{2 c \alpha^{(n)} n_{1}-n_{2}},
$$

where an initial guess can be computed e.g. as

$$
\alpha^{(0)}=x_{1} \text {. }
$$

The value of the penetration does not require an iterative procedure and can be computed after the definition of $\alpha$ as

$$
\xi^{3}=\frac{c \alpha^{2}-2 c \alpha x_{1}+x_{2}-n_{2} x_{1}}{2 c \alpha^{(n)} n_{1}-n_{2}} .
$$




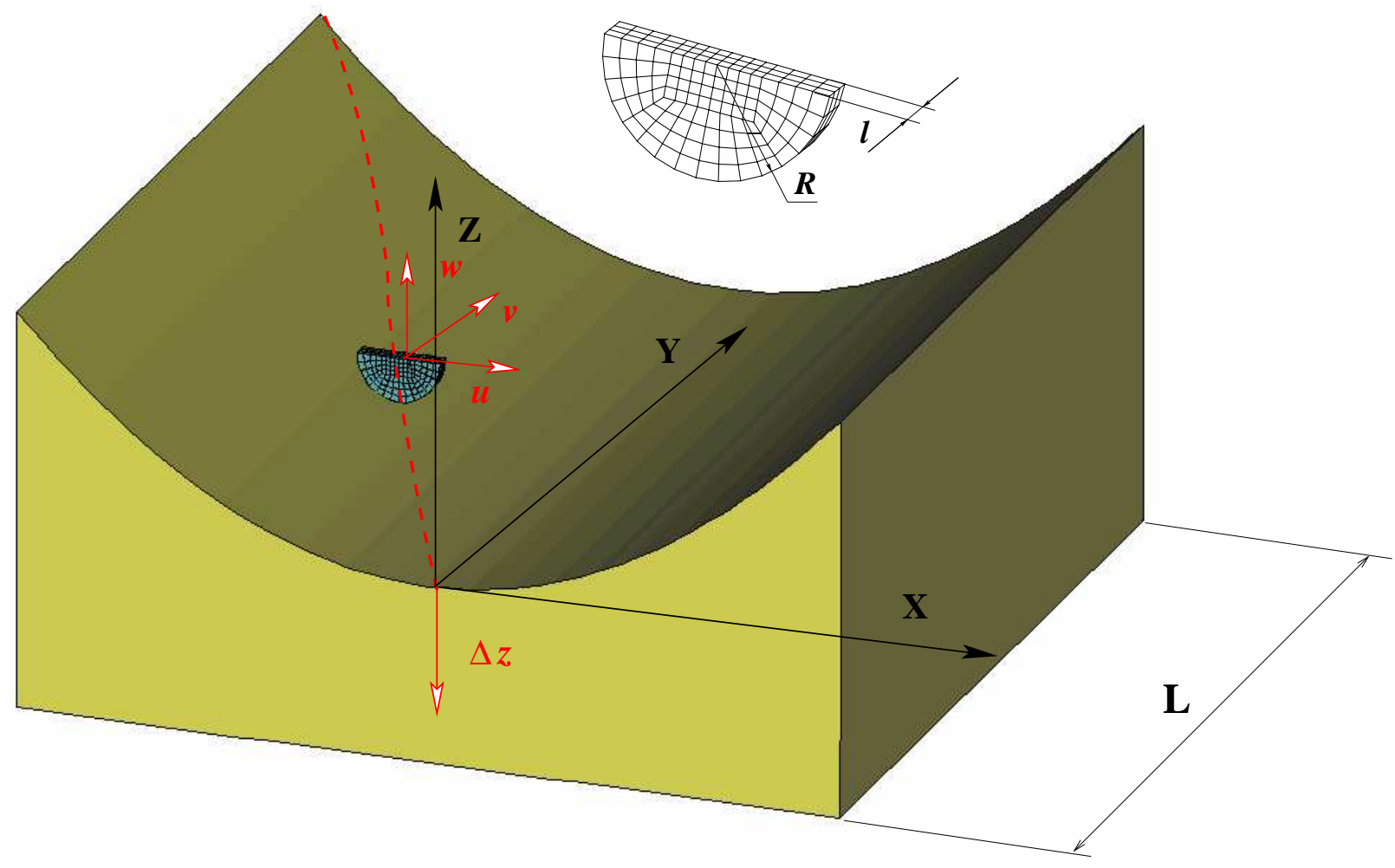

Figure 14: Spiral motion of a circular semi-cylinder on the parabolical cylinder. Segment-to-analytical surface approach.

Spiral equidistant motion to a cylinder. Now we consider a 3D motion on the surface of the parabolical cylinder. In order to generalize the equidistant motion in eqn. (116) into a spiral one we consider the parameterization in the form:

$$
x=v t, \quad y=H t / T,
$$

where $v$ is a loading rate, $T$ is the final load step. Thus, the spiral motion with the trajectory shown in Fig. 14 is defined as

$$
\mathbf{r}=\left\{\begin{array}{c}
v t(1-2 c h) \\
H t / T \\
h+c(v t)^{2}(1-2 c h)
\end{array}\right\}, \quad t=0,1,2, \ldots, T .
$$

For the numerical example, we chose the spiral motion of a short deformable circular semicylinder with radius $R=1$ and $l=0.4$ on the surface of the parabolical cylinder with parameters $c=5 \cdot 10^{-2}, H=20$, see Fig. 14. The semi-cylinder is made of the linear elastic material: $E=2.10 \cdot 10^{4} ; \nu=0.3$ and meshed with linear "solid-shell" elements $16 \times 3$ as shown in Fig. 14 . Coulomb friction with $\mu=0.3$ is specified between the bodies.

The loading process consists of two stages:

1) the circular semi-cylinder positioned at the initial point is pressed into the parabolical cylinder with $\Delta z=h=-0.01$ 
2) then the circular semi-cylinder is moving on the parabolical surface, providing an equidistant motion of the central axis with the distance $R+\Delta z=0.99$ according to eqn. (126) together with the upper surface parallel to the $\mathrm{X}-\mathrm{Z}$ plane. Thus, the central axis of the semi-cylinder is moving according to the following equation:

$$
\mathbf{r}=\left\{\begin{array}{c}
1.001 v t \\
20 t / T \\
h+5.005 \cdot 10^{-2}(v t)^{2}
\end{array}\right\}
$$

The displacements are applied in 1000 load steps with $v=10^{-2}$, providing an increment $\Delta y=0.02$ which is larger than the critical threshold value in eqn. (110). The Gauss integration formula with $4 \times 4$ integration points is used to check the value of penetration in eqn. (117). Here we concentrate again on the investigation of the influence of the various parts of the matrix on the convergence. Since the contact geometry is linear only due to the discretization of the semi-cylinder, the cases with the main matrix and the tangent matrix without curvature part are compared, see Table 5 for the first 20 loads steps. Obviously, it is definitely advantageous to use the tangent matrix without curvature parts, but keeping the rotational parts in this problem.

\begin{tabular}{|c|c|c|c|c|c|}
\hline \multicolumn{3}{|c|}{ Case 1 } & \multicolumn{3}{c|}{ Case 2 } \\
\hline No. l.s. & $\begin{array}{c}\text { No. } \\
\text { it./l.s. }\end{array}$ & $\begin{array}{c}\text { Cum. } \\
\text { No. it. }\end{array}$ & No. l.s. & $\begin{array}{c}\text { No. } \\
\text { it./l.s. }\end{array}$ & $\begin{array}{c}\text { Cum. } \\
\text { No. it. }\end{array}$ \\
\hline 1 & 9 & 9 & 1 & 10 & 10 \\
\hline 2 & 8 & 17 & $2-18$ & 13 & 231 \\
\hline 3 & 5 & 22 & 19 & 12 & 243 \\
\hline $4-20$ & 4 & 90 & 20 & 11 & 254 \\
\hline$\ldots$ & $\ldots$ & $\ldots$ & $\ldots$ & $\ldots$ & $\ldots$ \\
\hline
\end{tabular}

Table 5: Sliding of a semi-cylinder on a parabolical block. Bilinear contact elements. Segment-toanalytical surface contact approach. Influence of various contact stiffness parts on convergence. Case 1: excluding only curvature matrix; case 2: only main matrix. Comparison of no. of iterations for the fist 20 load steps (l.s.) 


\section{Conclusions}

In this contribution a fully convective description for frictional contact has been proposed. For this, a special local coordinate system according to the closest point procedure is used. The core of the description is to consider differential operations in the covariant form with expressing all values on the tangent plane. Thus, e.g. a penalty regularization of the Coloumb friction law leads to evolution equations expressed in the covariant derivatives. This approach has several advantages. First, the artificial non-symmetry of the sticking tangent matrix, which appeared in earlier publications, is removed. Second, the structure of each tangent matrix is more geometrical and algorithmic. It allows to distinguish between three parts of a tangent matrix, namely the "main" part, the "rotational" part and the "curvature" part. Further, the geometrical interpretation of the covariant derivatives leads to a continuous numerical integration algorithm which overcomes the discontinuities of the convective variables.

It was shown in the numerical examples that frictional contact problems can be subdivided into two types. The first type contains the development of a sticking-sliding zone. In this case, small loads steps, which can be estimated by considering an elastic layer under friction conditions, are necessary. In due course, it appears that then the differences in the convergence rate between computations with various tangent matrix are meaningless. For the second type a fully developed sliding is assumed and, therefore, fairly large steps beyond the threshold value can be applied. In this case it is important to compute with the matrix containing the rotational part. Keeping the curvature matrix leads only to a small improvement. 


\section{References}

[1] Al-Dojayli M., Meguid S.A. (2002) Accurate modeling of contact using cubic splines. Finite Elements in Analysis and Design, 38 pp. 337-352.

[2] Araki Y., Hjelmstad K. D. (2003) Rate-dependent projection operators for frictional contact constraints. International Journal For Numerical Methods in Engineering 57, pp. 923-954.

[3] Bonet J., Wood, R.D. (2000) Nonlinear continuum mechanics for finite element analysis. Cambridge: Cambridge Univ. Press.

[4] El-Abbasi N., Meguid S.A., Czekanski, A. (2001) On the modeling of smooth contact surfaces using cubic splines. International Journal for Numerical Methods in Engineering, 50, pp. 953967.

[5] Gray A. (1993) Modern differential geometry of curves and surfaces, Boca Raton: CRC Press.

[6] Harnau M., Konyukhov A., Schweizerhof K. Algorithmic aspects in large deformation contact analysis using "Solid-Shell" elements. Submitted for publication.

[7] Hauptmann R., Schweizerhof K. (1998) A systematic development of 'solid-shell' element formulation for linear and non-linear analysis employing only displacement degrees of freedom. International Journal for Numerical Methods in Engineering, 42, pp. 49-69.

[8] Hauptmann R., Doll S., Harnau M., Schweizerhof K. (2001) "Solid-shell" elements with linear and quadratic shape functions at large deformations with nearly incompressible materials. Computers \& Structures, 79, pp. 1671-1685.

[9] Kikuchi N., Oden, J. T. (1988) Contact problems in elasticity: a study of variational inequalities and finite element methods, Philadelphia: SIAM.

[10] Konyukhov A., Schweizerhof K. (2004) Contact formulation via a velocity description allowing efficiency improvements in frictionless contact analysis, Computational Mechanics, 33, pp. 165173.

[11] Krstulovic-Opara L., Wriggers P. (2001) Convergence studies for 2D smooth contact elements. ECCM-2001,European Conference on Computational Mechanics, Cracow, Poland.

[12] Krstulovic-Opara L., Wriggers P. (2002) A two-dimensional $C^{1}$-continuous contact element based on the moving friction cone description. WCCM V. Fifth World Congress on Computational Mechanics, Vienna, Austria.

[13] Krstulovic-Opara L., Wriggers P., Korelc J. (2002) A $C^{1}$-continuous formulation for 3D finite deformation frictional contact. Computational Mechanics, 29, pp. 27-42.

[14] Laursen T.A. (1992) Formulation and treatment of frictional contact problems using finite elements. PhD thesis, SUDAM. Report No. 92-6 Stanford University.

[15] Laursen T.A., Simo J.C. (1993) A continuum-based finite element formulation for the implicit solution of multibody large deformation frictional contact problems. International Journal for Numerical Methods in Engineering, 35, pp. 3451-3485, 1993.

[16] Laursen T.A., Padmanabhan V. (2001) A framework for development of surface smoothing procedures in large deformation frictional contact analysis. Finite Elements in Analysis and Design, 37, pp. 173-198. 
[17] Laursen T. A. (2002) Computational contact and impact mechanics. Fundamentals of modeling interfacial phenomena in nonlinear finite element analysis, Springer.

[18] Marsden J.E., Hughes T.J.R. (1983) Mathematical foundations of elasticity. Prentice-Hall, Inc.

[19] Parisch H., Lübbing Ch. (1997) A formulation of arbitrarily shaped surface elements for three-dimensional large deformation contact with friction. International Journal for Numerical Methods in Engineering, 40, pp. 3359-3383, 1997.

[20] Peric D., Owen D. R. J. (1992) Computational model for 3-D contact problems with friction based on the penalty method. International Journal for Numerical Methods in Engineering, 35, pp. 1289-1309.

[21] Pietrzak G., Curnier A. (1999) Large deformation frictional contact mechanics: continuum formulation and augmented Lagrangian treatment. Computer Methods in Applied Mechanics and Engineering, 177, pp. 351-381.

[22] Puso M.A., Laursen T.A. (2002) A 3D contact smoothing method using Gregory patches. International Journal For Numerical Methods in Engineering, 54, pp. 1161-1194.

[23] Schoen R., Yau S.-T. (1994) Lectures on differential geometry. Boston, International Press.

[24] Simo J.C., Laursen T.A. (1992) An Augmented Lagrangian treatment of contact problems involving friction. Computers and Structures, 42, pp. 97-116.

[25] Simo J.C., Hughes T.J.R. (1992) Elastoplasticity and Viscoplasticity: computational aspects. Springer-Verlag, Berlin.

[26] Stadler M., Holzapfel G.A., Korelc J. (2003) $C^{n}$-continuous modeling of smooth contact surfaces using NURBS and application to 2D problems. International Journal for Numerical Methods in Engineering, 57, pp. 2177-2203.

[27] Taylor R.L. (1987) FEAP - A Finite Element Analysis Program. University of California at Berkeley, Berkeley. Schweizerhof, K. and Coworkers, FEAP - Mechanik - Karlsruhe, Institut für Mechanik, Universität Karlsruhe.

[28] Wriggers P., Vu Van, Stein E. (1990) Finite element formulation of large deformation impactcontact problem with friction. Computers and Structures, 37, pp. 319-331.

[29] Wriggers P. (1995) Finite element algorithm for contact problems. Archives of Computational Methods in Engineering, 2(4), pp. 1-49.

[30] Wriggers P., Krstulovic-Opara L., Korelc J. (2001) Smooth $C^{1}$-interpolations for twodimensional frictional contact problems International Journal for Numerical Methods in Engineering, 51, pp. 1469-1495.

[31] Wriggers P. (2002) Computational Contact Mechanics. John Wiley \& Sons.

[32] Zhang, H., Zhong W., Gu Y. (1998) A combined parametric quadratic programming and iteration method for 3-D elastic-plastic frictional contact problem analysis. Computer methods in applied mechanics and engineering, vol. 155, pp. 307-324. 
[33] Zhang, H. W., Xu, W. L., Di, S. L., Thomson, P. F. (2002) Quadratic programming method in numerical simulation of metal forming process. Computer methods in applied mechanics and engineering, vol. 191, pp. 5555-5578.

[34] Zhong Zhi-Hua (1993) Finite element procedures for contact-impact problems. Oxford, Oxford Univ. Press. 


\section{APPENDIX}

\section{A. Prove of the Weingarten formula.}

Having taken the derivative of the unity equation $\mathbf{n} \cdot \mathbf{n}=1$ with respect to surface coordinates $\xi^{i}$, we obtain $\mathbf{n} \cdot \mathbf{n}_{i}=0$, from which follows that the vectors $\mathbf{n}_{i}$ are orthogonal to $\mathbf{n}$ and, therefore, lay on the tangent plane of the surface. Thus, $\mathbf{n}_{i}$ is expressed as a sum of the surface vectors $\boldsymbol{\rho}_{i}$

$$
\mathbf{n}_{i}=c_{i .}^{k} \boldsymbol{\rho}_{k}
$$

Computing a dot product with $\boldsymbol{\rho}_{j}$

$$
\left(\mathbf{n}_{i} \cdot \boldsymbol{\rho}_{j}\right)=c_{i .}^{k}\left(\boldsymbol{\rho}_{k} \cdot \boldsymbol{\rho}_{j}\right) \rightarrow\left(\mathbf{n}_{i} \cdot \boldsymbol{\rho}_{j}\right)=c_{i .}^{k} a_{k j},
$$

a derivative of the orthogonality condition $\boldsymbol{\rho}_{i} \cdot \mathbf{n}=0$, gives $\boldsymbol{\rho}_{i j} \cdot \mathbf{n}+\boldsymbol{\rho}_{i} \cdot \mathbf{n}_{j}=0$. Thus

$$
h_{i j} \equiv\left(\boldsymbol{\rho}_{i j} \cdot \mathbf{n}\right)=-\left(\boldsymbol{\rho}_{i} \cdot \mathbf{n}_{j}\right) .
$$

Therefore, the $c_{i .}^{k}$ can be defined as

$$
c_{i .}^{k}=-h_{i j} a^{j k},
$$

from which Weingarten's formula (13) is obtained.

\section{B. Prove of the Gauss-Kodazzi formula.}

In general, the derivatives of the coordinate surface vectors $\boldsymbol{\rho}_{i}$ are no longer on the surface, therefore, one should express them by the vectors $\boldsymbol{\rho}_{1}, \boldsymbol{\rho}_{2}, \mathbf{n}$

$$
\boldsymbol{\rho}_{i j}=\Gamma_{i j}^{k} \boldsymbol{\rho}_{k}+h_{i j} \mathbf{n} .
$$

Expressions for $\Gamma_{i j}^{k}$ and $h_{i j}$ follow from the computation of the dot product with the basis vectors $\boldsymbol{\rho}_{j}$ and the normal $\mathbf{n}$.

\section{Covariant derivative of covariant components.}

In the case of covariant components we need the derivative of a contravariant base vector $\frac{\partial \boldsymbol{\rho}^{i}}{\partial \xi^{j}}$ instead of $\boldsymbol{\rho}_{i j}$, see eqn. (17). First, take the derivative of the mixed metric components:

$$
\frac{\partial a_{i}^{k}}{\partial \xi^{j}}=\frac{\partial\left(\boldsymbol{\rho}_{i} \cdot \boldsymbol{\rho}^{k}\right)}{\partial \xi^{j}}=\left(\boldsymbol{\rho}_{i j} \cdot \boldsymbol{\rho}^{k}\right)+\left(\boldsymbol{\rho}_{i} \cdot \frac{\partial \boldsymbol{\rho}^{k}}{\partial \xi^{j}}\right)=\Gamma_{i j}^{k}+\left(\boldsymbol{\rho}_{i} \cdot \boldsymbol{\rho}_{, j}^{k}\right)=0
$$

therefore,

$$
\left(\boldsymbol{\rho}_{i} \cdot \boldsymbol{\rho}_{, j}^{k}\right)=-\Gamma_{i j}^{k}
$$


and the covariant derivative for the covariant component gets the following form

$$
\nabla_{j} T_{i}=\frac{\partial T_{i}}{\partial \xi^{j}}-T_{k} \Gamma_{i j}^{k}
$$

\section{Prove that the full time derivative is a Lie time derivative.}

In order to prove eqn. (32) consider the vector $\mathbf{r}\left(\xi^{i}\right)$ in the reference Cartesian frame:

$$
\mathbf{r}=X^{k} \mathbf{e}_{k}
$$

where $\mathbf{e}_{k}$ are unit vectors of the Cartesian reference frame. By definition of the reference frame the vectors $\mathbf{e}_{k}$ are time independent. Since here only the spatial case is considered, all indices are running from 1 to 3 . The vector $\mathbf{r}$ is assumed to be time independent only for simplicity without loss of generality. The coordinate vectors $\mathbf{r}_{i}$ are defined as

$$
\mathbf{r}_{i}=\frac{\partial X^{k}}{\partial \xi^{i}} \mathbf{e}_{k}=\left(F^{-1}\right)_{i}^{k} \mathbf{e}_{k}
$$

where $\left(F^{-1}\right)_{i}^{k}$ are components of the inverse gradient deformation tensor $\mathbf{F}$ with components

$$
F_{j}^{i}=\frac{\partial \xi^{i}}{\partial X^{j}}
$$

which are used for the vice versa transformation:

$$
\mathbf{e}_{k}=F_{k}^{i} \mathbf{r}_{i}
$$

Eqns. (139) and (137) give the push-forward operator $\mathbf{F}$ and the pull-back operator $\mathbf{F}^{-1}$ respectively in a tensor form:

$$
\mathbf{F}=F_{j}^{i} \mathbf{r}_{i} \otimes \mathbf{e}^{j}, \quad \mathbf{F}^{-1}=\left(F^{-1}\right)_{j}^{i} \mathbf{e}_{i} \otimes \mathbf{r}^{j} .
$$

The Lie time derivative of the spatial vector $\mathbf{T}=T^{i} \mathbf{r}_{i}$ is taken following the rule: pull-back to the reference configuration, take time derivative, push-forward to the current configuration:

$$
\begin{aligned}
\mathcal{L}_{t} \mathbf{T}=\mathbf{F} \frac{d}{d t}\left(\mathbf{F}^{-1} \mathbf{T}\right) & = \\
=F_{n}^{k} \mathbf{r}_{k} \otimes \mathbf{e}^{n} \cdot \frac{d}{d t}\left(\left(F^{-1}\right)_{i}^{j} \mathbf{e}_{j} \otimes \mathbf{r}^{i} \cdot T^{m} \mathbf{r}_{m}\right) & =F_{n}^{k} \mathbf{r}_{k} \otimes \mathbf{e}^{n} \cdot \frac{d}{d t}\left(\left(F^{-1}\right)_{i}^{j} \mathbf{e}_{j} \delta_{m}^{i} T^{m}\right)= \\
& =F_{n}^{k} \mathbf{r}_{k} \delta_{j}^{n} \frac{d}{d t}\left(\left(F^{-1}\right)_{i}^{j} T^{i}\right)=\frac{d\left(\left(F^{-1}\right)_{i}^{j} T^{i}\right)}{d t} F_{j}^{k} \mathbf{r}_{k} .
\end{aligned}
$$

This is a full time derivative. It can be seen directly

$$
\frac{d\left[\left(F^{-1}\right)_{i}^{j} T^{i}\right]}{d t} F_{j}^{k} \mathbf{r}_{k}=\frac{d\left[\left(F^{-1}\right)_{i}^{j} T^{i}\right]}{d t} \mathbf{e}_{j}=\frac{d\left[\left(F^{-1}\right)_{i}^{j} T^{i} \mathbf{e}_{j}\right]}{d t}=\frac{d\left[T^{i} \mathbf{r}_{i}\right]}{d t}=\frac{d \mathbf{T}}{d t} .
$$

For the prove only the time independence of the reference basis vectors $\mathbf{e}_{j}$ was used. 
Some algebraic manipulations of equation (141) are required to show this for the components.

$$
\begin{gathered}
\mathcal{L}_{t} \mathbf{T}=\frac{d\left[\left(F^{-1}\right)_{i}^{j} T^{i}\right]}{d t} F_{j}^{k} \mathbf{r}_{k}=\frac{\partial T^{i}}{\partial t}\left(F^{-1}\right)_{i}^{j} F_{j}^{k} \mathbf{r}_{k}+\frac{\partial T^{i}}{\partial \xi^{n}}\left(F^{-1}\right)_{i}^{j} F_{j}^{k} \dot{\xi}^{n} \mathbf{r}_{k}+\frac{\partial\left(F^{-1}\right)_{i}^{j}}{\partial \xi^{n}} T^{i} F_{j}^{k} \dot{\xi}^{n} \mathbf{r}_{k}=(143) \\
=\frac{\partial T^{i}}{\partial t} \delta_{i}^{k} \mathbf{r}_{k}+\frac{\partial T^{i}}{\partial \xi^{n}} \delta_{i}^{k} \dot{\xi}^{n} \mathbf{r}_{k}+\frac{\partial\left(F^{-1}\right)_{i}^{j}}{\partial \xi^{n}} T^{i} F_{j}^{k} \dot{\xi}^{n} \mathbf{r}_{k} .
\end{gathered}
$$

The last term contains Christoffel symbols. In order to elaborate this, their determination in the reference frame has to be considered. Equation (15) can be written as

$$
\Gamma_{i j}^{k} a_{k l}=\mathbf{r}_{i j} \cdot \mathbf{r}_{l},
$$

or in Cartesian coordinates as

$$
\Gamma_{i j}^{k} \frac{\partial X^{m}}{\partial \xi^{k}} \frac{\partial X^{m}}{\partial \xi^{l}}=\frac{\partial X^{n}}{\partial \xi^{i} \partial \xi^{j}} \frac{\partial X^{n}}{\partial \xi^{l}}
$$

and, exploiting the chain rule,

$$
\Gamma_{i j}^{k} \frac{\partial X^{m}}{\partial \xi^{k}} \frac{\partial X^{m}}{\partial \xi^{l}} \frac{\partial \xi^{k}}{\partial X^{p}} \frac{\partial \xi^{l}}{\partial X^{r}}=\frac{\partial X^{n}}{\partial \xi^{i} \partial \xi^{j}} \frac{\partial X^{n}}{\partial \xi^{l}} \frac{\partial \xi^{k}}{\partial X^{p}} \frac{\partial \xi^{l}}{\partial X^{r}} \rightarrow \Gamma_{i j}^{k} \delta_{p}^{m} \delta_{r}^{m}=\frac{\partial X^{n}}{\partial \xi^{i} \partial \xi^{j}} \delta_{r}^{n} \frac{\partial \xi^{k}}{\partial X^{p}}
$$

finally one obtains

$$
\Gamma_{i j}^{k}=\frac{\partial X^{n}}{\partial \xi^{i} \partial \xi^{j}} \frac{\partial \xi^{k}}{\partial X^{n}}
$$

Now the Lie derivative (eqn. (143)) can be written as:

$$
\mathcal{L}_{t} \mathbf{a}==\frac{\partial a^{i}}{\partial t} \mathbf{r}_{i}+\frac{\partial a^{i}}{\partial \xi^{n}} \dot{\xi}^{n} \mathbf{r}_{i}+\frac{\partial X^{j}}{\partial \xi^{i} \partial \xi^{n}} \frac{\partial \xi^{k}}{\partial X^{j}} a^{i} \dot{\xi}^{n} \mathbf{r}_{k}=\frac{\partial a^{i}}{\partial t} \mathbf{r}_{i}+\frac{\partial a^{i}}{\partial \xi^{n}} \dot{\xi}^{n} \mathbf{r}_{i}+\Gamma_{i j}^{k} \dot{\xi}^{j} a^{i} \mathbf{r}_{k}
$$

This is a full vector derivative (18) including the covariant derivative (19). 


\section{Contents}

1 Introduction 1

2 Geometry and Kinematics of Contact 2

2.1 Local surface coordinate system and its geometrical characteristics . . . . . . . . 3

2.1.1 The fundamental tensors and property of the contact surface . . . . . . . 4

2.1.2 Differential operations in the surface coordinate system . . . . . . . . . . 5

2.2 Spatial coordinate system and its characteristics . . . . . . . . . . . . . 6

2.2.1 Projection of the contact point vector onto the master surface. . . . . . . 6

2.2.2 Spatial local coordinate system. Geometrical characteristics on the tangent plane. ............................ 7

2.2.3 Motion of a slave point. Convective velocity on the tangent plane. . . . . . 9

2.3 Geometrical interpretation of covariant derivative and numerical realization . . . 10

2.3.1 Continuous numerical integration algorithm for a relative motion vector $\Delta \boldsymbol{\rho} 11$

2.3.2 Parallel translation of a vector $\mathbf{T}$ on the tangent plane. . . . . . . . . . . 12

3 Weak form for finite element formulation and regularized contact conditions 13

3.1 Weak formulation in the spatial coordinate system. . . . . . . . . . . . . . . . . 13

3.2 Regularization by the penalty method . . . . . . . . . . . . . . . . . . . . . 14

3.2.1 Normal contact conditions. . . . . . . . . . . . . . . . . . . . 14

3.2.2 Tangential contact conditions. Evolution equations. . . . . . . . . . . 15

4 Consistent linearization $\quad 18$

4.1 Linearization of the normal contact expression . . . . . . . . . . . . . . . . . 18

4.2 Linearization of the tangential contact expression . . . . . . . . . . . . . . . . . . . 18

4.2.1 Linearization of $\delta \xi^{i} \ldots \ldots$. . . . . . . . . . . . . . . . . . . . . . . . . . . . . . . . . . . . . . . . . . . .

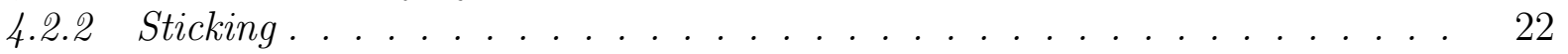

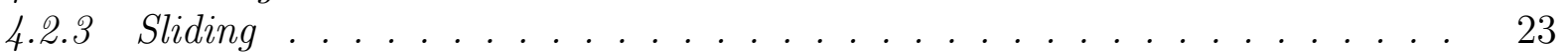

5 Global solution scheme. Summary of the results. 25

6 Numerical examples 28

6.1 Sliding of a block with a linear approximation of the contact surfaces. . . . . . . . 28

6.2 Sliding of a block. Quadratical approximation of the contact surfaces. . . . . . . . 32

6.3 Large sliding on a rigid parabolical cylinder . . . . . . . . . . . . . . . . . . . . 36

6.3.1 Contact with a surface described by analytical functions . . . . . . . . . . 36

7 Conclusions $\quad 41$

8 APPENDIX $\quad 45$ 